\title{
Synaptic plasticity and NO-cGMP-PKG signaling coordinately regulate ERK-driven gene expression in the lateral amygdala and in the auditory thalamus following Pavlovian fear conditioning
}

\author{
Kristie T. Ota, ${ }^{1}$ Melissa S. Monsey, ${ }^{1}$ Melissa S. Wu, ${ }^{1}$ Grace J. Young, ${ }^{1}$ and \\ Glenn E. Schafe ${ }^{1,2,3}$ \\ ${ }^{7}$ Department of Psychology, Yale University, New Haven, Connecticut 06520, USA; ${ }^{2}$ Interdepartmental Neuroscience Program, \\ Yale University, New Haven, Connecticut 06520, USA
}

\begin{abstract}
We have recently hypothesized that NO-cGMP-PKG signaling in the lateral nucleus of the amygdala (LA) during auditory fear conditioning coordinately regulates ERK-driven transcriptional changes in both auditory thalamic (MGm/PIN) and LA neurons that serve to promote pre- and postsynaptic alterations at thalamo-LA synapses, respectively. In the present series of experiments, we show that $\mathrm{N}$-methyl-D-aspartate receptor (NMDAR)-driven synaptic plasticity and NO-cGMP-PKG signaling in the LA regulate the training-induced expression of ERK and the ERK-driven immediate early genes (IEGs) Arc/ Arg3.1, c-Fos, and EGR-1 in the LA and the MGm/PIN. Rats receiving intra-LA infusion of the NR2B selective antagonist Ifenprodil, the NOS inhibitor 7-Ni, or the PKG inhibitor Rp-8-Br-PET-cGMPS exhibited significant decreases in ERK activation and in the training-induced expression of all three IEGs in the LA and MGm/PIN while intra-LA infusion of the PKG activator 8-Br-cGMP had the opposite effect. Remarkably, those rats given intra-LA infusion of the membrane impermeable NO scavenger c-PTIO exhibited significant decreases in ERK activation and ERK-driven IEG expression in the $\mathrm{MGm} / \mathrm{PIN}$, but not in the LA. Together with our previous experiments, these results suggest that synaptic plasticity and the NO-cGMP-PKG signaling pathway promote fear memory consolidation, in part, by regulating ERK-driven transcription in both the LA and the MGm/PIN. They further suggest that synaptic plasticity in the LA during fear conditioning promotes ERK-driven transcription in MGm/PIN neurons via NO-driven "retrograde signaling."
\end{abstract}

[Supplemental material is available online at http://www.learnmem.org.]

Most recent research aimed at studying fear memory formation and synaptic plasticity in the amygdala has focused on $\mathrm{N}$-methyl-D-aspartate receptor (NMDAR)-mediated alterations in intracellular signaling pathways in lateral amygdala (LA) neurons that are thought, in part, to promote long-term plastic change and memory through the alterations of transcription and translation (Schafe et al. 2001; Lin et al. 2003). However, while the LA has clearly been established as an essential locus of fear memory formation and consolidation (Blair et al. 2001; Schafe et al. 2001, 2005a; Rodrigues et al. 2004a), recent studies have suggested that the auditory thalamus (including the medial division of the medial geniculate nucleus and the posterior intralaminar nucleus; MGm/PIN) may also play a critical role in fear memory consolidation. For example, recent work in our laboratory and others has pointed to a critical role of ERK and ERK-driven transcription in fear memory consolidation not only in the LA (Schafe et al. 2000, 2005a), but also in the MGm/PIN (Apergis-Schoute et al. 2005; Parsons et al. 2006; Han et al. 2008; Overeem et al. 2010). Interestingly, recent findings suggest that the functional significance of ERK-driven gene expression in the MGm/PIN may be to promote presynaptic aspects of fear memory formation back at the level of the LA. For example,

\footnotetext{
${ }^{3}$ Corresponding author.

E-mail glenn.schafe@yale.edu; fax (203) 432-7172.

Article is online at http://www.learnmem.org/cgi/doi/10.1101/Im.1592510.
}

activation of ERK/MAPK in the MGm/PIN is required for synaptic plasticity in the LA (Apergis-Schoute et al. 2005), and ERK-driven gene expression in MGm/PIN neurons regulates the expression of presynaptically localized proteins at LA synapses following fear learning (Overeem et al. 2010).

Based in part on these findings, our laboratory has recently developed a revised model of fear memory consolidation that emphasizes the importance of ERK-driven gene expression on both sides of the thalamo-LA synapse. Further, we propose that synaptic plasticity and nitric oxide (NO) signaling in the LA during fear learning may be critical for coordinating ERK-driven transcriptional changes in both the MGm/PIN and the LA that may, in turn, contribute to pre- and postsynaptic aspects of plasticity back in the LA. We have recently shown that NO-cGMP-PKG signaling in the LA is critical for fear memory consolidation (Schafe et al. 2005b; Ota et al. 2008), an effect that is attributable, at least in part, to activation of the ERK/MAPK signaling pathway (Schafe et al. 2005b; Ota et al. 2008). The NO-cGMP-PKG signaling pathway is known to activate various components of the MAPK signaling pathway upstream of and including ERK (Lander et al. 1995, 1996, 1997; Yun et al. 1998, 1999), and to have a variety of effects on synaptic plasticity both pre- and postsynaptically. In vitro studies, for example, have shown that NO-cGMP-PKG signaling may promote the activation of protein kinase signaling cascades in the postsynaptic cell, leading to activation of transcription and translation that are critical for long-term synaptic plasticity 
and memory formation (Lu et al. 1999; Chien et al. 2003). In addition, NO has also been proposed to act as a "retrograde messenger" that may be critical for promoting mobilization of synaptic vesicles in the presynaptic cell, leading to enhanced transmitter release (Ninan et al. 2006), as well as structural changes in the presynaptic terminal (Antonova et al. 2001; Wang et al. 2005).

In the present study, we use pharmacological and immunolabeling methods to examine whether NMDAR-driven synaptic plasticity and NO-cGMP-PKG signaling in the LA regulate both ERK and ERK-driven gene expression in LA and MGm/PIN neurons following auditory fear conditioning. Our findings suggest that synaptic plasticity and the NO-cGMP-PKG signaling pathway in the LA regulate fear memory consolidation, in part, by promoting ERK-driven transcriptional regulation in both the LA and the MGm/PIN. They further suggest that NO-driven "retrograde signaling" in the LA during fear conditioning promotes ERK activation and ERK-driven transcription in MGm/PIN neurons.

\section{Results}

\section{Synaptic plasticity and the NO-cGMP-PKG signaling pathway in the LA regulate phosphorylation of ERK/ MAPK in the LA and MGm/PIN following fear conditioning}

We have recently shown that blockade or facilitation of the NO-cGMP-PKG signaling pathway in the LA impairs or enhances memory consolidation of Pavlovian fear conditioning and synaptic plasticity in the LA by activating the ERK/MAPK signaling pathway, suggesting that NO-driven increases in ERK-driven transcriptional regulation in the LA regulate the formation of long-term memory storage (Ota et al. 2008). Previous work in our laboratory and others, however, has pointed to a critical role of ERK and ERK-mediated transcription in fear memory consolidation not only in the LA, but also in the auditory thalamus (Apergis-Schoute et al. 2005). Specifically, intra-MGm/PIN blockade of ERK activation or mRNA synthesis have been shown to impair memory consolidation of an auditory fear memory (Apergis-Schoute et al. 2005; Parsons et al. 2006). Conversely, overexpression of the transcription factor CREB in the MGm/ PIN has been shown to enhance fear memory formation (Han et al. 2008). Furthermore, recent findings showing that inhibition of ERK signaling in the MGm/PIN impairs LTP in the LA have suggested that the functional significance of ERK-driven transcription in the MGm/PIN is to promote presynaptic aspects of plasticity in the LA (Apergis-Schoute et al. 2005). When considered collectively with our recent observations that NO signaling in the LA is required for fear memory consolidation (Schafe et al. 2005b; Ota et al. 2008), we have hypothesized that synaptic plasticity and NO signaling in the LA at the time of fear learning coordinate ERK-driven transcriptional changes in MGm/PIN and LA neurons that serve to promote pre- and postsynaptic alterations at thalamo-LA synapses. In the present series of experiments, we have used pharmacological and Western blotting techniques to examine whether synaptic plasticity and NO signaling in the LA coordinately regulate phosphorylation of ERK/ MAPK in the LA and MGm/PIN following fear conditioning.

\section{Synaptic plasticity and NO signaling in the LA selectively regulate phosphorylation of ERK/MAPK in the LA and MGm/PIN following fear conditioning}

In our first experiment, we asked whether synaptic plasticity and NO signaling in the LA regulate ERK/MAPK phosphorylation in the LA and MGm/PIN following fear conditioning (Fig. 1). In these experiments, rats were given intra-LA infusion of either vehicle, the NR2B selective antagonist Ifenprodil $(1 \mu \mathrm{g} /$ side; $0.5 \mu \mathrm{L})$, the NOS inhibitor $7-\mathrm{Ni}(1 \mu \mathrm{g} / \mathrm{side} ; 0.5 \mu \mathrm{L})$, or the membrane impermeable NO scavenger c-PTIO $(1 \mu \mathrm{g} /$ side; $0.5 \mu \mathrm{L}$ ), followed by evaluation of the effects of each of these pharmacological manipulations on training-induced ERK activation in both LA and MGm/PIN. This dose of Ifenprodil has previously been shown to significantly impair fear memory acquisition when infused into the LA prior to fear conditioning (Rodrigues et al. 2001). The doses of c-PTIO and 7-Ni have been shown to significantly impair fear memory consolidation when infused into the LA prior to fear conditioning; that is, fear acquisition and short-term memory (STM) are intact, while long-term memory (LTM) is impaired (Schafe et al. 2005b). Thirty minutes following infusion, rats were trained with three tone-shock pairings and sacrificed $1 \mathrm{~h}$ after training (Fig. 1A), a time point that we have previously shown to be optimal for observing training-induced increases in ERK activation in the LA (Schafe et al. 2000). Western blotting on LA and MGm/PIN homogenates was performed to determine whether training-induced activation of ERK/MAPK in the LA and MGm/PIN is impaired following intra-LA infusion of the NR2B antagonist, the NOS inhibitor, or the NO scavenger.

The effects of intra-LA infusion of Ifenprodil, 7-Ni, and c-PTIO on training-induced ERK activation in the LA are depicted in Figure 1D, while representative Western blots can be viewed in Figure 1B. Relative to vehicle controls, rats given intra-LA infusions of either Ifenprodil or 7-Ni exhibited marked reductions in ERK activation in LA homogenates, while rats infused with c-PTIO did not. The ANOVA (drug by kinase) revealed a significant effect for drug (vehicle vs. Ifenprodil vs. 7-Ni vs. c-PTIO; $F_{(3,56)}=$ $11.73, P<0.001)$, a nonsignificant effect for kinase, and a nonsignificant drug by kinase interaction. Specifically, post-hoc tests revealed that ERK labeling in the vehicle-infused group differed significantly from that infused with either Ifenprodil $(P<0.001)$ or 7-Ni $(P<0.001)$, but did not significantly differ from the group infused with c-PTIO $(P>0.05)$. In addition, ERK labeling in the c-PTIO group differed significantly from that in both Ifenprodil $(P<0.001)$ and $7-\mathrm{Ni}(P<0.001)$ groups. Furthermore, this impairment of ERK activation cannot be accounted for by differences in total ERK/MAPK. Total ERK/MAPK levels, expressed relative to the loading control GAPDH, were not significantly changed from vehicle levels following infusion of any of these drugs (Fig. 1E). The ANOVA for total ERK revealed a nonsignificant effect for drug, a nonsignificant effect for kinase, and a nonsignificant drug by kinase interaction.

The effects of intra-LA infusion of Ifenprodil, 7-Ni, and c-PTIO on training-induced ERK activation in the MGm/PIN are depicted in Figure 1F, while representative Western blots can be viewed in Figure 1C. Relative to vehicle controls, rats given intra-LA infusions of either Ifenprodil, 7-Ni, or c-PTIO exhibited marked reductions in ERK activation in MGm/PIN homogenates. The ANOVA (drug by kinase) revealed a significant effect for drug (vehicle vs. Ifenprodil vs. $7-\mathrm{Ni}$ vs. c-PTIO; $F_{(3,56)}=10.00, P<$ 0.001), a nonsignificant effect for kinase, and a nonsignificant drug by kinase interaction (Fig. 1F). Specifically, post-hoc tests revealed that the vehicle condition differed significantly from all three drug conditions (Ifenprodil $[P<0.001]$, 7-Ni $[P<$ $0.001]$, and c-PTIO $[P<0.05])$. Furthermore, this impairment of ERK activation cannot be accounted for by differences in total ERK/MAPK (Fig. 1G). Total ERK/MAPK levels, expressed relative to the loading control GAPDH, were not significantly changed from vehicle levels following infusion of any of these drugs. The ANOVA revealed nonsignificant effects for drug, kinase, and the drug by kinase interaction.

Thus, intra-LA infusion of agents that block synaptic plasticity (Ifenprodil) or nNOS activation (7-Ni) impair training-induced 

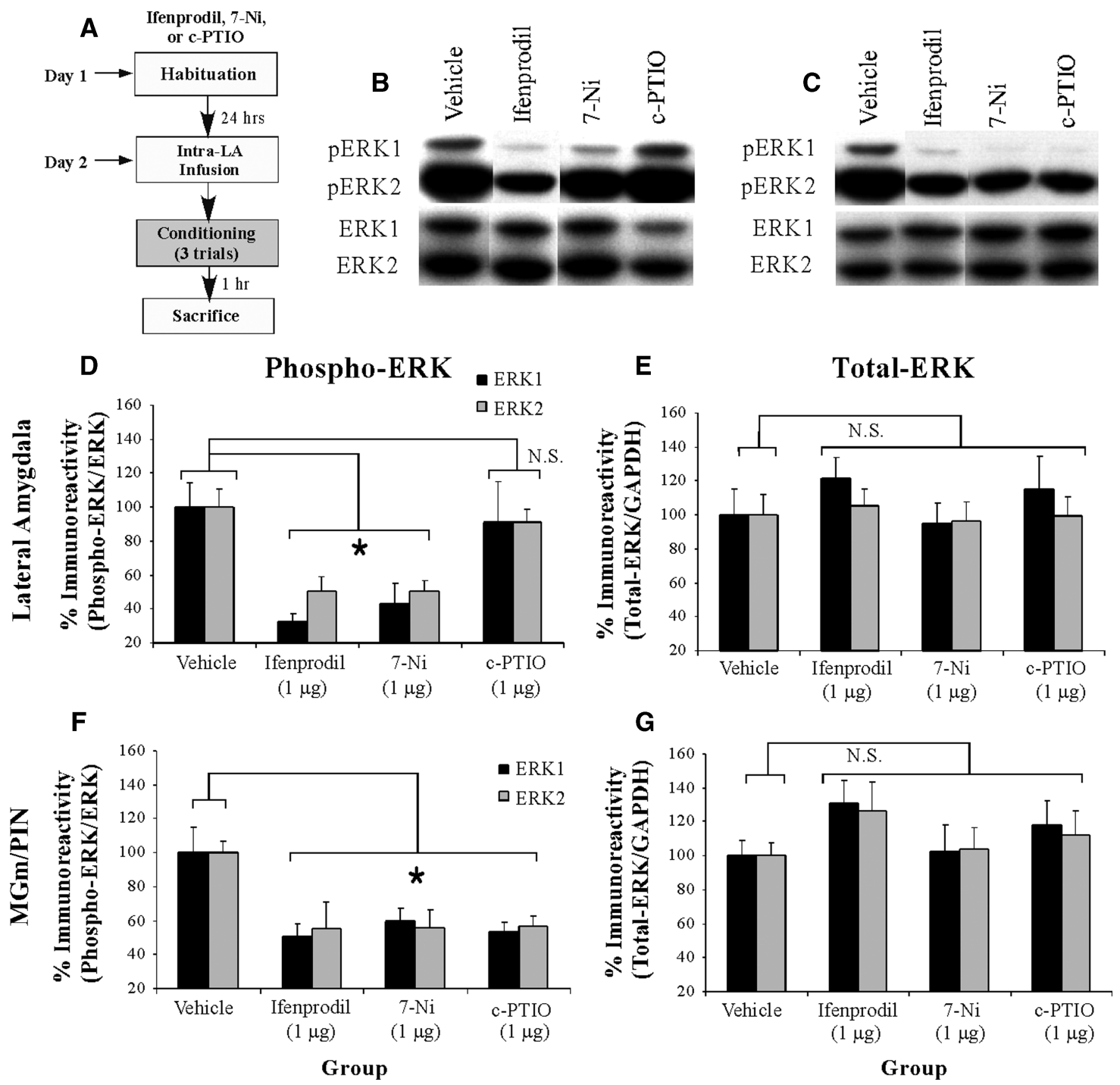

Figure 1. Synaptic plasticity and NO signaling in the LA selectively regulate phosphorylation of ERK/MAPK in the LA and MGm/PIN following fear conditioning. (A) Schematic of behavioral protocol. Rats were given intra-LA infusion of the vehicle $(n=8)$, the NR2B selective antagonist Ifenprodil $(1 \mu \mathrm{g} /$ side; $n=8)$, the NOS inhibitor 7-Ni $(1 \mu \mathrm{g} / \mathrm{side} ; n=8)$, or the membrane impermeable NO scavenger c-PTIO $(1 \mu \mathrm{g} / \mathrm{side} ; n=8)$, followed 30 min later by fear conditioning. Rats were sacrificed $1 \mathrm{~h}$ following training. (B) Representative blots for both phospho-ERK (pERK) and total ERK in the LA. (C) Representative blots for both pERK and total ERK in the MGm/PIN. (D) Mean ( \pm SEM) percent pERK immunoreactivity from LA punches taken from rats given intra-LA infusions of vehicle, Ifenprodil, 7-Ni, or c-PTIO. Here, pERK levels have been normalized to total ERK levels for each sample. (E) Mean ( \pm SEM) percent total-ERK immunoreactivity from LA punches taken from rats given intra-LA infusions of vehicle, Ifenprodil, 7-Ni, or C-PTIO. Here, total ERK levels have been normalized to GAPDH levels for each sample. $(F)$ Mean ( \pm SEM) percent pERK immunoreactivity from MGm/PIN punches taken from the rats in D given intra-LA infusions of vehicle, Ifenprodil, 7-Ni, or C-PTIO. Here, pERK levels have been normalized to total ERK levels for each sample. $(G)$ Mean ( \pm SEM) percent total-ERK immunoreactivity from MGm/PIN punches taken from the rats in $D$ given intra-LA infusions of vehicle, Ifenprodil, 7-Ni, or c-PTIO. Here, total ERK levels have been normalized to GAPDH levels for each sample. $\left({ }^{*}\right) P<0.05$ relative to vehicle-infused rats.

ERK-activation in both the LA and the MGm/PIN, while intra-LA infusion of a membrane impermeable NO scavenger (c-PTIO) selectively impairs ERK activation in the MGm/PIN.

\section{Intra-LA infusion of a PKG inhibitor impairs ERK phosphorylation in the $L A$ and $M G m / P I N$ following fear conditioning}

In our next series of experiments, we looked further downstream in the NO-cGMP-PKG signaling pathway to examine the effect of inhibition of the cGMP-dependent protein kinase (PKG) in the LA on ERK activation in the LA and MGm/PIN using a combination of Western blotting and immunohistochemistry. Rats were given intra-LA infusion of either vehicle or the PKG inhibitor Rp-8-Br-PET-cGMPS $(1 \mu \mathrm{g} /$ side; $0.5 \mu \mathrm{L})$, a dose that we have recently shown to be effective in impairing fear memory consolidation (Ota et al. 2008). One hour following infusion, rats were trained with three tone-shock pairings and then sacrificed $1 \mathrm{~h}$ later (Fig. 2A). Western blotting and immunohistochemistry were performed to determine whether training-induced activation of ERK/MAPK in the LA and MGm/PIN is impaired following intra-LA infusion of the PKG inhibitor. 
The effect of intra-LA infusion of Rp-8-Br-PET-cGMPS on ERK activation in the LA is depicted in Figure 2D, while representative Western blots can be viewed in Figure 2B. Relative to vehicleinfused controls, rats given intra-LA infusion of Rp-8-Br-PETcGMPS prior to training exhibited significant decreases in both phospho-ERK1 and phospho-ERK2 in the LA (Fig. 2D). The ANOVA revealed a significant effect for drug (vehicle vs. Rp-8-BrPET-cGMPS; $\left.F_{(1,20)}=17.98, P<0.001\right)$, a nonsignificant effect for kinase, and a nonsignificant drug by kinase interaction. Furthermore, this impairment of ERK activation cannot be accounted for by differences in total ERK/MAPK (Fig. 2E). Total ERK/MAPK levels, expressed relative to the loading control GAPDH, were not significantly changed from vehicle levels following infusion of Rp-8-Br-PET-cGMPS. The ANOVA revealed nonsignificant effects for drug, kinase, and the drug by kinase interaction.

The effect of intra-LA infusion of Rp-8-Br-PET-cGMPS on ERK activation in the MGm/PIN is depicted in Figure 2F, while representative Western blots can be viewed in Figure 2C. Relative to vehicle-infused controls, rats given intra-LA infusions of the PKG inhibitor Rp-8-Br-PET-cGMPS prior to training exhibited significant decreases in both phospho-ERK1 and phospho-ERK2 in the MGm/PIN (Fig. 2F). The ANOVA revealed a significant effect for drug (vehicle vs. Rp-8-Br-PET-cGMPS; $F_{(1,22)}=16.59, P<$ 0.001), a nonsignificant effect for kinase, and a nonsignificant drug by kinase interaction. Furthermore, this impairment of ERK activation cannot be accounted for by differences in total ERK/MAPK (Fig. 2G). Total ERK/MAPK levels, expressed relative to the loading control GAPDH, were not significantly changed from vehicle levels following infusion of Rp-8-Br-PET-cGMPS. The ANOVA revealed nonsignificant effects for drug, kinase, and the drug by kinase interaction.

Representative sections from the immunohistochemical experiments are depicted in Figure $2 \mathrm{H}-\mathrm{K}$. Similar to the findings of previous studies (Schafe et al. 2000; Paul et al. 2007), pERK-labeled cells in vehicle-infused rats were evident in the ventral regions of the LA and the basal nucleus of the amygdala, as well as the central nucleus (CE) and the amygdala-striatal transition zone (AST; Fig. 2H). In the $\mathrm{MGm} / \mathrm{PIN}$, we observed pERK-labeled cells throughout the PIN and extending dorsally into the MGm/PIN. In contrast, little to no pERK labeling was observed in the MGv (Fig. 2J). Consistent with the findings of the Western blotting experiments, we observed reductions in pERK-labeled cells in the LA (Fig. 2I) and MGm/PIN (Fig. 2K) following intra-LA infusion of Rp-8-Br-PET-cGMPS. Cell counts of pERK-labeled cells in the LA and MGm/PIN for rats infused with Rp-8-Br-PET-cGMPS can be viewed in Supplemental Figure 1A,B, while statistical analysis of these data may be viewed in Supplemental Analysis 1.
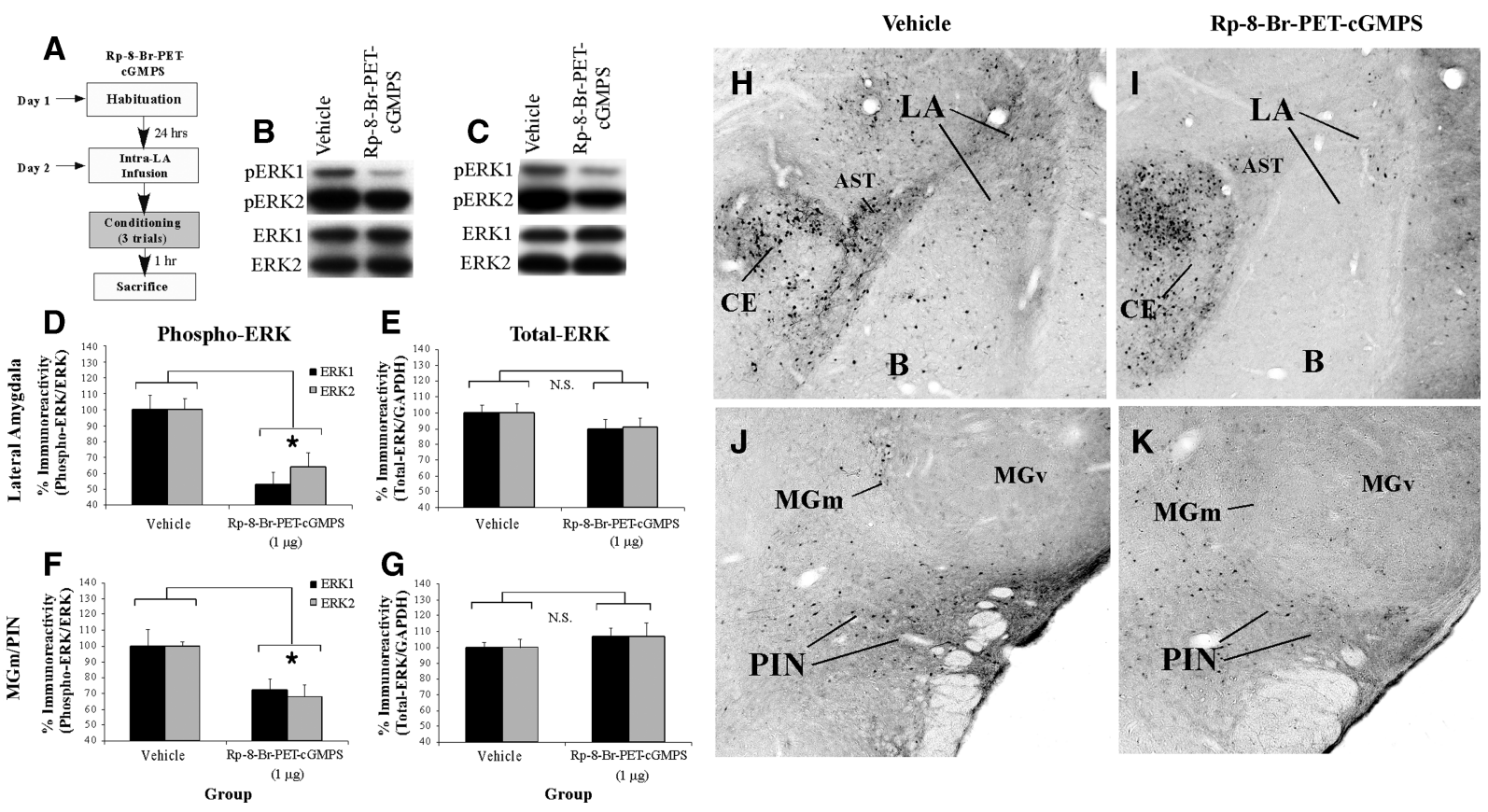

Figure 2. Intra-LA infusion of a PKG inhibitor impairs ERK phosphorylation in the LA and MGm/PIN following fear conditioning. ( $A$ ) Schematic of behavioral protocol. Rats were given intra-LA infusion of the vehicle $(n=7)$ or Rp-8-Br-PET-cGMPS $(1 \mu \mathrm{g} / \mathrm{side} ; n=5)$, followed $1 \mathrm{~h}$ later by fear conditioning. Rats were sacrificed $1 \mathrm{~h}$ following training. (B) Representative blots for both phospho-ERK ( $p$ ERK) and total ERK in the LA. (C) Representative blots for both pERK and total ERK in the MGm/PIN. (D) Mean ( \pm SEM) percent pERK immunoreactivity from LA punches taken from rats given intra-LA infusions of vehicle or Rp-8-Br-PET-cGMPS. Here, pERK levels have been normalized to total ERK levels for each sample. (E) Mean ( \pm SEM) percent total-ERK immunoreactivity from LA punches taken from rats given intra-LA infusions of vehicle or Rp-8-Br-PET-cGMPS. Here, total ERK levels have been normalized to GAPDH levels for each sample. $(F)$ Mean $( \pm$ SEM) percent pERK immunoreactivity from MGm/PIN punches taken from the rats in $D$ given intra-LA infusions of vehicle $(n=6)$ or Rp-8-Br-PET-cGMPS $(1 \mu \mathrm{g} / \mathrm{side} ; n=7)$. Here, pERK levels have been normalized to total ERK levels for each sample. $(G)$ Mean ( \pm SEM) percent total-ERK immunoreactivity from MGm/PIN punches taken from rats in $D$ given intra-LA infusions of vehicle or Rp-8-Br-PET-cGMPS Here, total ERK levels have been normalized to GAPDH levels for each sample. $\left(^{*}\right) P<0.05$ relative to vehicle-infused rats. $(H)$ Representative $10 X$ photomicrograph of the LA of an animal given intra-LA infusion of vehicle, trained, and sacrificed $1 \mathrm{~h}$ later. (I) Representative 10X photomicrograph of the LA of an animal given intra-LA infusion of Rp-8-Br-PET-cGMPS $(1 \mu \mathrm{g} /$ side), trained, and sacrificed $1 \mathrm{~h}$ later. $(J)$ Representative 10X photomicrograph of the $\mathrm{MGm} / \mathrm{PIN}$ of an animal given intra-LA infusion of vehicle, trained, and sacrificed $1 \mathrm{~h}$ later. $(K)$ Representative $10 \mathrm{X}$ photomicrograph of the MGm/PIN of an animal given intra-LA infusion of Rp-8-Br-PET-cGMPS $(1 \mu \mathrm{g} / \mathrm{side})$, trained, and sacrificed $1 \mathrm{~h}$ later. (LA) Lateral nucleus of the amygdala; (CE) central nucleus of the amygdala; (B) basal nucleus of the amygdala; (AST) amygdala-striatal transition zone; (MGm) medial division of the medial geniculate nucleus; (PIN) posterior intralaminar nucleus; (MGv) ventral division of the medial geniculate nucleus. 


\section{Intra-LA infusion of a PKG activator enhances ERK phosphorylation} in the LA and MGm/PIN

The previous experiment examined the effects of intra-LA inhibition of the PKG signaling pathway on ERK activation in the LA and $\mathrm{MGm} / \mathrm{PIN}$. In the present experiment, we examined the effect of intra-LA infusion of a PKG activator on ERK activation in the LA and MGm/PIN (Fig. 3). In this experiment, rats were given intra-LA infusion of the PKG activator 8-Br-cGMP $(10 \mu \mathrm{g} /$ side; $0.5 \mu \mathrm{L}$ ), a dose that we have previously shown to be effective in enhancing fear memory consolidation (Ota et al. 2008). One hour following the infusion, rats were trained with two toneshock pairings and then sacrificed $1 \mathrm{~h}$ later (Fig. 3A). We have previously used this slightly weaker two-pairing training protocol in behavioral experiments to allow for observation of memory enhancements in the 8-Br-cGMP-infused group relative to vehicle-infused controls (Ota et al. 2008). As before, Western blotting and immunohistochemistry were performed to determine whether training-induced activation of ERK/MAPK in the LA and MGm/PIN is enhanced following intra-LA infusion of the PKG activator.

The effect of intra-LA infusion of 8-Br-cGMP on ERK activation in the LA is depicted in Figure 3D, while representative Western blots can be viewed in Figure 3B. Relative to vehicleinfused controls, rats given intra-LA infusion of 8-Br-cGMP prior to training exhibited significant increases in both phosphoERK1 and phospho-ERK2 in the LA (Fig. 3D). The ANOVA revealed a significant effect for drug (vehicle vs. 8-Br-cGMP; $F_{(1,24)}=31.44$, $P<0.001$ ), a nonsignificant effect for kinase, and a nonsignificant drug by kinase interaction. Furthermore, this increase in ERK activation cannot be accounted for by differences in total ERK/MAPK (Fig. 3E). Total ERK/MAPK levels, expressed relative to the loading control GAPDH, were not significantly changed from vehicle levels following infusion of 8-Br-cGMP. The ANOVA revealed a nonsignificant effect for drug, a nonsignificant effect for kinase, and a nonsignificant drug by kinase interaction.

The effect of intra-LA infusion of 8-Br-cGMP on ERK activation in the MGm/PIN is depicted in Figure 3F, while representative Western blots can be viewed in Figure 3C. Relative to vehicleinfused controls, rats given intra-LA infusions of the PKG activator 8-Br-cGMP prior to training exhibited significant increases in both phospho-ERK1 and phospho-ERK2 in the MGm/PIN (Fig. 3F). The ANOVA revealed a significant effect for drug (vehicle vs. 8-BrcGMP; $\left.F_{(1,24)}=12.35, P<0.01\right)$, a nonsignificant effect for kinase, and a nonsignificant drug by kinase interaction. Furthermore, this increase in ERK activation cannot be accounted for by differences in total ERK/MAPK (Fig. 3G). Total ERK/MAPK levels, expressed relative to the loading control GAPDH, were not significantly changed from vehicle levels following infusion of 8-Br-cGMP. The ANOVA revealed nonsignificant effects for drug, kinase, and the drug by kinase interaction.

Representative sections from the immunohistochemical experiments are depicted in Figure $3 \mathrm{H}-\mathrm{K}$. Consistent with the
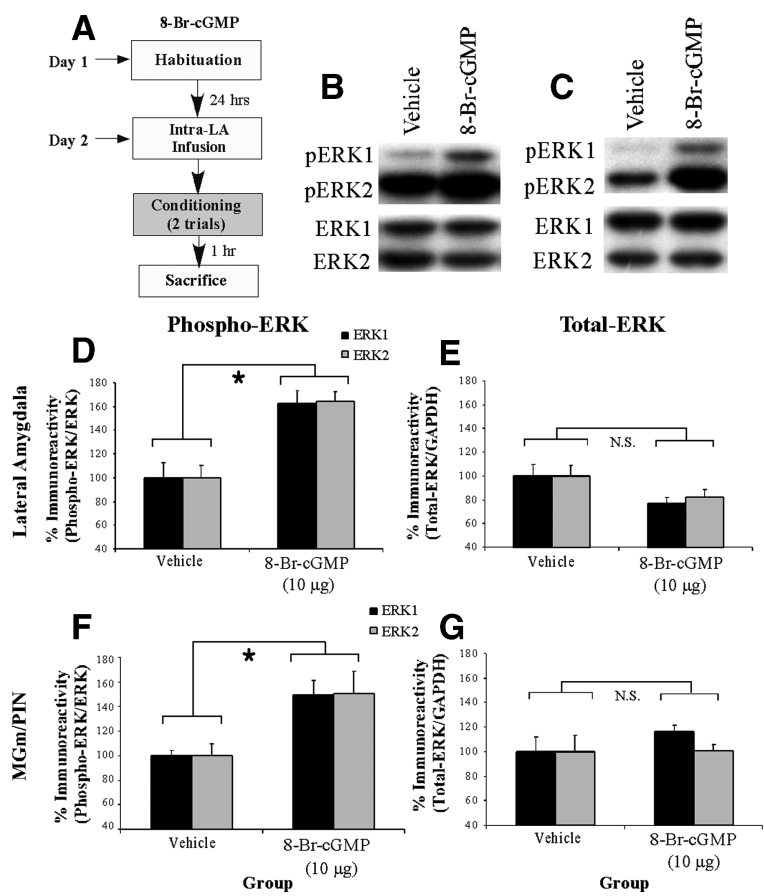

- ERK1
口ERK2
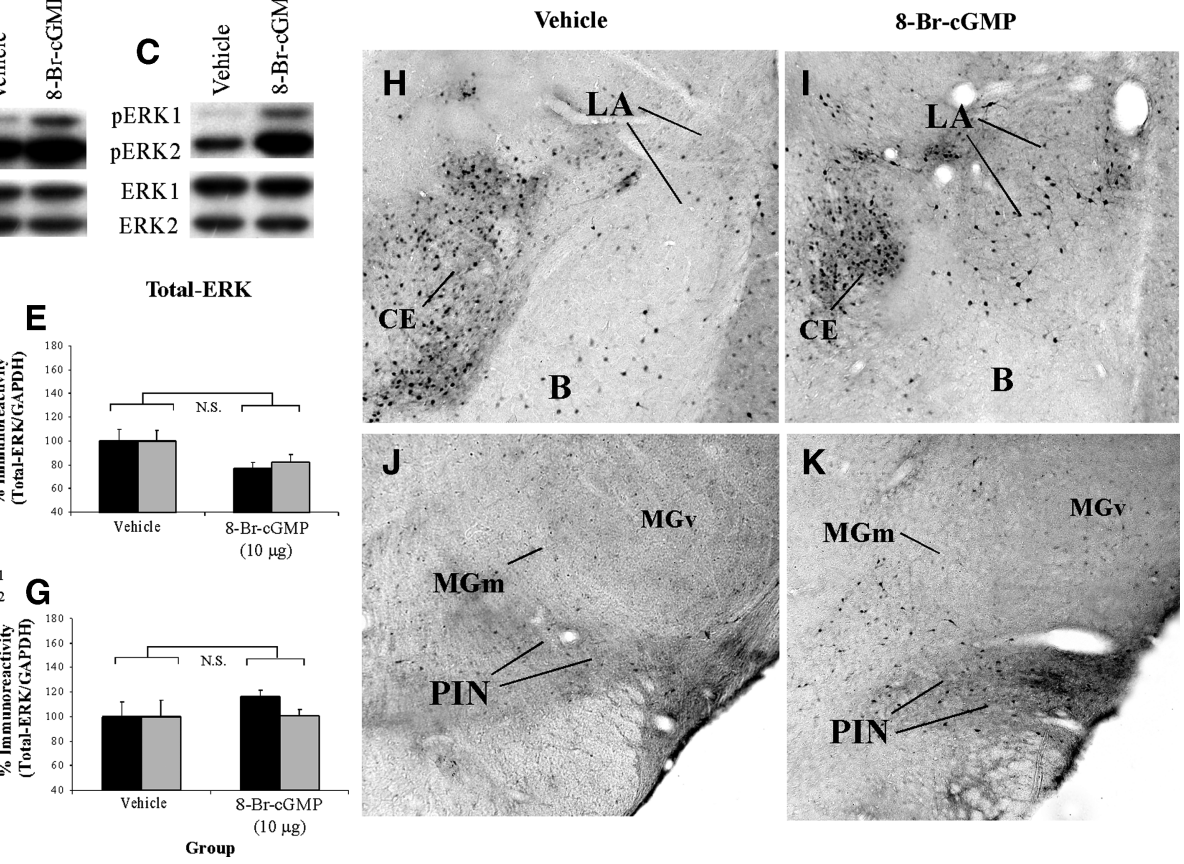

Figure 3. Intra-LA infusion of a PKG activator enhances ERK phosphorylation in the LA and MGm/PIN following fear conditioning. (A) Schematic of behavioral protocol. Rats were given intra-LA infusion of the vehicle $(n=6)$ or 8 -Br-cGMP $(10 \mu \mathrm{g} / \mathrm{side} ; n=8)$, followed $1 \mathrm{~h}$ later by fear conditioning. Rats were sacrificed $1 \mathrm{~h}$ following training. (B) Representative blots for both phospho-ERK (pERK) and total ERK in the LA. (C) Representative blots for both pERK and total ERK in the MGm/PIN. (D) Mean ( \pm SEM) percent pERK immunoreactivity from LA punches taken from rats given intra-LA infusions of vehicle or 8-Br-cGMP. Here, pERK levels have been normalized to total ERK levels for each sample. (E) Mean ( \pm SEM) percent total-ERK immunoreactivity from LA punches taken from rats given intra-LA infusions of vehicle or 8-Br-cGMP. Here, total ERK levels have been normalized to GAPDH levels for each sample. $(F)$ Mean ( \pm SEM) percent pERK immunoreactivity from MGm/PIN punches taken from the rats in $D$ given intra-LA infusions of vehicle or 8-Br-cGMP. Here, pERK levels have been normalized to total ERK levels for each sample. $(G)$ Mean $( \pm S E M)$ percent total-ERK immunoreactivity from $\mathrm{MGm} / \mathrm{PIN}$ punches taken from the rats in $D$ given intra-LA infusions of vehicle or 8-Br-cGMP. Here, total ERK levels have been normalized to GAPDH levels for each sample. $(H)$ Representative 10X photomicrograph of the LA of an animal given intra-LA infusion of vehicle, trained, and sacrificed $1 \mathrm{~h}$ later. (I) Representative 10X photomicrograph of the LA of an animal given intra-LA infusion of 8-Br-cGMP (10 $\mu \mathrm{g} /$ side), trained, and sacrificed $1 \mathrm{~h}$ later. (J) Representative 10X photomicrograph of the MGm/PIN of an animal given intra-LA infusion of vehicle, trained, and sacrificed $1 \mathrm{~h}$ later. $(K)$ Representative 10X photomicrograph of the MGm/PIN of an animal given intra-LA infusion of $8-\mathrm{Br}$ cGMP $(10 \mu \mathrm{g} /$ side), trained, and sacrificed $1 \mathrm{~h}$ later. $(*) P<0.05$ relative to vehicle-infused rats. 
findings of the Western blotting experiments, we observed elevations in pERK-labeled cells in the LA (Fig. 3I) and MGm/PIN (Fig. 3K) following intra-LA infusion of 8-Br-cGMP. Relative to vehicle-infused controls (Fig. 3H,J), most of the enhanced pERK labeling in 8-Br-cGMP-infused rats was evident in ventral regions of the LA (Fig. 3I), while that in the MGm/PIN was observed throughout the PIN and extending dorsally into the MGm/PIN (Fig. 3K). As before, little to no pERK labeling was observed in the MGv (Fig. 3K). Cell counts of pERK-labeled cells in the LA and MGm/PIN in rats infused with 8 -Br-cGMP can be viewed in Supplemental Figure 1C,D, while statistical analysis of these data may be viewed in Supplemental Analysis 1 .

Together with our previous experiments, these findings suggest that NMDAR-driven synaptic plasticity and NO-cGMP-PKG signaling in the LA promotes fear memory consolidation, in part, by regulating ERK signaling in both the LA and the MGm/ PIN. Further, given the fact that the NOS inhibitor 7-Ni impairs ERK activation in both LA and MGm/PIN, while the membrane impermeable NO scavenger c-PTIO selectively impairs ERK activation in the MGm/PIN, our findings suggest that synaptic plasticity in the LA during fear conditioning promotes ERK/MAPK activation in MGm/PIN neurons via extracellular release of NO from LA neurons.

\section{Synaptic plasticity and the NO-cGMP-PKG signaling pathway in the LA regulate ERK-driven IEG expression in the LA and MGm/PIN following fear conditioning}

In our first series of experiments, we showed that NMDAR-driven synaptic plasticity and NO-cGMP-PKG signaling in the LA regulate ERK/MAPK phosphorylation in the LA and MGm/PIN. In this next series of experiments, we examined whether these same signaling pathways also regulate downstream targets of ERK, including the immediate early genes (IEGs) Arc/Arg3.1, c-Fos, and EGR-1, in the LA and the MGm/PIN. Each of these downstream targets has been implicated in memory formation, including fear memory formation (Rosen et al. 1998; Malkani and Rosen 2000; Hall et al. 2001; Malkani et al. 2004; Ko et al. 2005; Zhang et al. 2005; Huff et al. 2006), but little is known about whether they are regulated by NO signaling in the LA and MGm/PIN.

\section{Synaptic plasticity and NO signaling in the LA selectively regulate ERK-driven} IEG expression in the LA and MGm/PIN following fear conditioning

In the first series of experiments, we asked whether NMDARdriven synaptic plasticity and NO signaling in the LA regulate ERK-driven IEG expression in the LA after fear conditioning (Fig. 4). We gave rats intra-LA infusion of either vehicle, Ifenprodil $(1 \mu \mathrm{g} /$ side; $0.5 \mu \mathrm{L}), 7$-Ni $(1 \mu \mathrm{g} /$ side; $0.5 \mu \mathrm{L})$, or c-PTIO $(1 \mu \mathrm{g} /$ side; $0.5 \mu \mathrm{L})$. Thirty minutes following infusion, rats were conditioned using three tone-shock pairings, then sacrificed at $2 \mathrm{~h}$ following training, a time point that previous studies have determined to be effective for observing training-induced regulation of IEGs in both LA and MGm/PIN (Wallace et al. 1998; Ploski et al. 2008) (Fig. 4A). Western blotting was used to probe for Arc/Arg3.1, c-Fos, and EGR-1 in LA and MGm/PIN homogenates.

The effects of intra-LA infusion of Ifenprodil, 7-Ni, and c-PTIO on IEG expression in the LA are depicted in Figure 4D, while representative Western blots can be viewed in Figure 4B. Relative to vehicle controls, rats given intra-LA infusion of either Ifenprodil or 7-Ni prior to training exhibited significant reductions in levels of Arc/Arg3.1, c-Fos, and EGR-1 immunoreactivity in LA homogenates, while rats infused with c-PTIO did not (Fig. 4D). The ANOVAs for Arc/Arg3.1 $\left(F_{(3,22)}=3.26, P<0.05\right)$,
c-Fos $\left(F_{(3,24)}=6.28, P<0.01\right)$, and EGR-1 $\left(F_{(3,24)}=5.82, P<\right.$ $0.01)$ all revealed significant overall effects. Specifically, post-hoc $t$-tests revealed that for each IEG the vehicle group differed significantly from those infused with Ifenprodil (Arc: $P<0.05$; c-Fos: $P<0.01$; EGR-1: $P<0.05$ ) and 7-Ni (Arc: $P<0.05$; c-Fos: $P<0.05$; EGR-1: $P<0.05)$, but not from the group infused with c-PTIO (Arc: $P>0.05$; c-Fos: $P>0.05$; EGR-1: $P>0.05$ ). Furthermore, IEG expression in the group infused with c-PTIO differed significantly from that infused with Ifenprodil (c-Fos: $P<$ 0.01; EGR-1: $P<0.01$ ) and 7-Ni (c-Fos: $P<0.05$; EGR-1: $P<$ 0.01 ) for c-Fos and EGR-1. Importantly, levels of the loading control, GAPDH, did not differ between any of the groups for Arc/Arg3.1, c-Fos, and EGR-1.

The effects of intra-LA infusion of Ifenprodil, 7-Ni, and c-PTIO on IEG expression in the MGm/PIN are depicted in Figure $4 \mathrm{E}$, while representative Western blots can be viewed in Figure 4C. Relative to vehicle controls, rats given intra-LA infusion of either Ifenprodil, 7-Ni, or c-PTIO prior to training exhibited significant reductions in the expression of all three IEGs in the MGm/PIN (Fig. 4E). The ANOVAs for Arc/Arg3.1 $\left(F_{(3,27)}=\right.$ $16.09, P<0.001)$, c-Fos $\left(F_{(3,27)}=5.02, P<0.01\right)$, and EGR-1 $\left(F_{(3,27)}=11.85, \quad P<0.001\right)$ all revealed significant effects. Specifically, post-hoc $t$-tests revealed that the vehicle-infused group differed significantly from that infused with Ifenprodil (Arc: $P<0.001$; c-Fos: $P<0.01$; EGR-1: $P<0.001$ ), 7-Ni (Arc: $P<0.001$; c-Fos: $P<0.01$; EGR-1: $P<0.001$ ), and c-PTIO (Arc: $P<0.001$; c-Fos: $P<0.05$; EGR-1: $P<0.001)$ for all three IEGs. In addition, levels of the loading control, GAPDH, did not differ between any of the groups for Arc, c-Fos, and EGR-1.

Importantly, the reduction in IEG expression in both the LA and MGm/PIN following intra-LA infusion of either Ifenprodil, 7-Ni, or c-PTIO was not observed in naïve animals that did not receive fear conditioning (Fig. 4F-J). Representative Western blots from either LA or MGm/PIN for naïve rats given intra-LA infusion of Ifenprodil, 7-Ni, or c-PTIO can be viewed in Figure 4, G and $\mathrm{H}$, respectively. Rats given intra-LA infusions of vehicle, Ifenprodil, 7-Ni, or c-PTIO prior to sacrifice at the same time as the trained animals described above exhibited no significant differences in levels of Arc/Arg3.1, c-Fos, or EGR-1 protein in the LA (Fig. 4I). In addition, overall protein levels remained unchanged, as the levels of the loading control, GAPDH, did not differ between any of the groups for the three IEGs. Similarly, naïve rats given intra-LA infusion of Ifenprodil, 7-Ni, or c-PTIO exhibited no significant differences in levels of Arc/Arg3.1, c-Fos, and EGR-1 protein in the MGm/PIN (Fig. 4J). In addition, levels of the loading control, GAPDH, did not differ between any of the groups for the three IEGs. Collectively, our findings suggest that the reductions in IEG expression in the LA and MGm/PIN following intra-LA infusion of Ifenprodil, 7-Ni, or c-PTIO cannot be attributed to infusion of these drugs alone, but rather by infusion in combination with fear conditioning.

\section{Intra-LA infusion of a PKG inhibitor or a PKG activator impairs or enhances, respectively, ERK-driven IEG expression in the LA and $M G m / P I N$ following fear conditioning}

In our next series of experiments, we examined the effects of either inhibition or activation of PKG in the LA on training-induced IEG expression in the LA and MGm/PIN. As in our initial experiments examining ERK activation, rats were given intra-LA infusion of either Rp-8-Br-PET-cGMPS $(1 \mu \mathrm{g} /$ side; $0.5 \mu \mathrm{L}), 8$-Br-cGMP $(10 \mu \mathrm{g} /$ side; $0.5 \mu \mathrm{L})$, or their respective vehicle solutions $1 \mathrm{~h}$ prior to fear conditioning, followed by sacrifice $2 \mathrm{~h}$ later (Figs. 5A, 6A). Next, samples taken from the LA and $\mathrm{MGm} / \mathrm{PIN}$ of these animals were probed using Western blotting for Arc/Arg3.1, c-Fos, and EGR-1. 


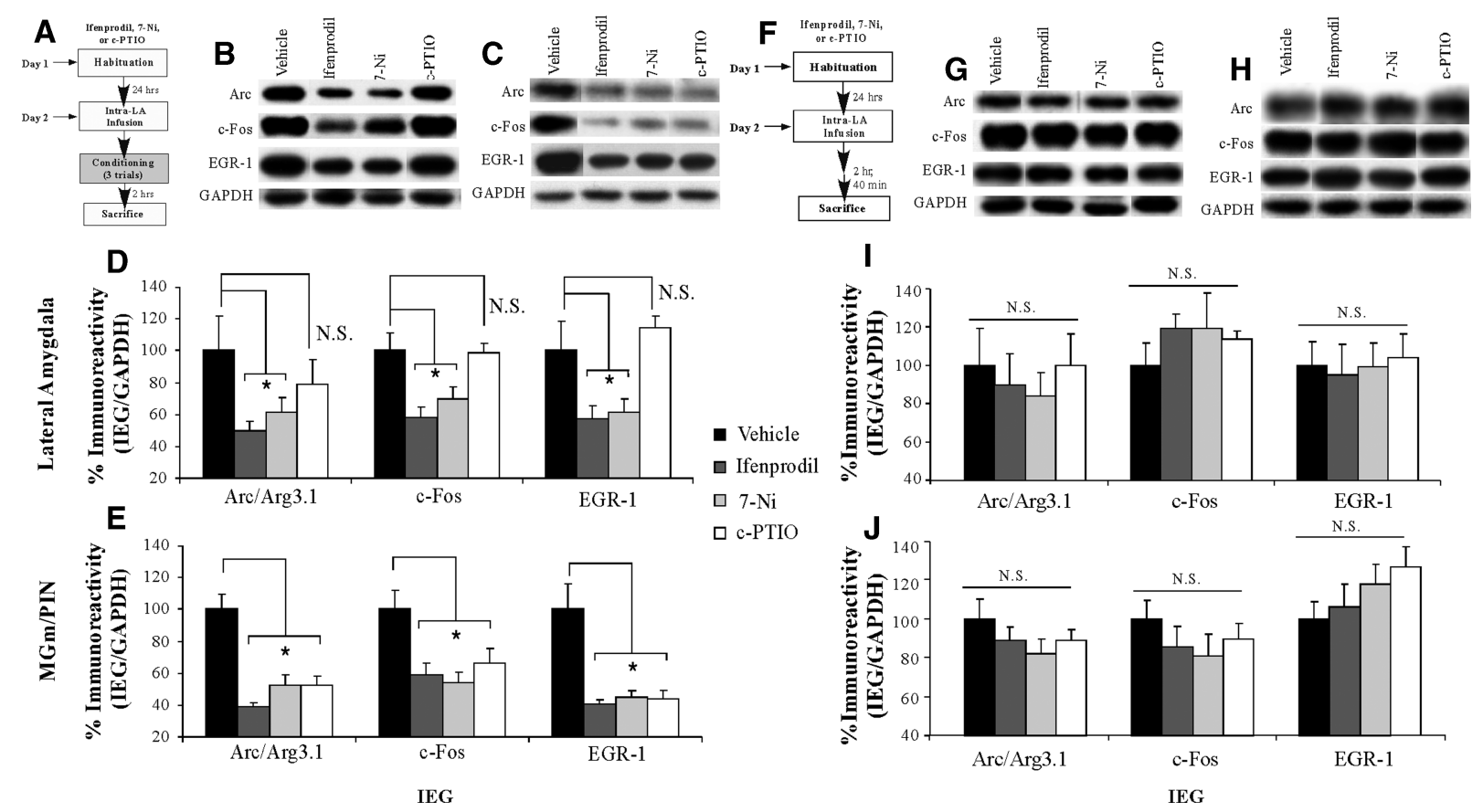

Figure 4. Synaptic plasticity and NO signaling in the LA selectively regulate ERK-driven IEG expression in the LA and MGm/PIN following fear conditioning. (A) Schematic of behavioral protocol. Rats were given intra-LA infusion of the vehicle, Ifenprodil ( $1 \mu \mathrm{g} / \mathrm{side}), 7-\mathrm{Ni}$ ( $1 \mu \mathrm{g} / \mathrm{side})$, or c-PTIO (1 $\mu \mathrm{g} / \mathrm{side})$, followed $30 \mathrm{~min}$ later by fear conditioning. Rats were sacrificed $2 \mathrm{~h}$ following training. (B) Representative blots for Arc/Arg3.1, C-Fos, EGR-1, and GAPDH in the LA. (C) Representative blots for Arc/Arg3.1, c-Fos, EGR-1, and GAPDH in the MGm/PIN. (D) Mean ( \pm SEM) percent Arc/Arg3.1, c-Fos, and EGR-1 immunoreactivity from LA punches taken from rats given intra-LA infusions of vehicle (Arc/Arg3.1: $n=7$, c-Fos: $n=8$, EGR-1: $n=8$ ), Ifenprodil (Arc/ Arg3.1: $n=6$, c-Fos: $n=6$, EGR-1: $n=6$ ), 7-Ni (Arc/Arg3.1: $n=7$, c-Fos: $n=7$, EGR-1: $n=7$ ), or c-PTIO (Arc/Arg3.1: $n=6$, c-Fos: $n=7$, EGR-1: $n=7)$. Here, Arc/Arg3.1, C-Fos, and EGR-1 levels have been normalized to GAPDH levels for each sample. (E) Mean ( \pm SEM) percent Arc/Arg3.1, c-Fos, and EGR-1 immunoreactivity from MGm/PIN punches taken from rats in $D$ given intra-LA infusions of vehicle (Arc/Arg3.1: $n=8, \mathrm{c}-$ Fos: $n=8$, EGR-1: $n=8$ ), Ifenprodil (Arc/Arg3.1: $n=8, \mathrm{c}-$ Fos: $n=8$, EGR-1: $n=8$ ), 7-Ni (Arc/Arg3.1: $n=7$, c-Fos: $n=7$, EGR-1: $n=7$ ), or c-PTIO (Arc/Arg3.1: $n=8$, c-Fos: $n=8$, EGR-1: $n=8$ ). Here, Arc/Arg3.1, c-Fos, and EGR-1 levels have been normalized to GAPDH levels for each sample. ( $F$ ) Schematic of behavioral protocol. Rats were given intra-LA infusion of the vehicle $(n=7)$, Ifenprodil $(1 \mu \mathrm{g} / \mathrm{side} ; n=7)$, $7-\mathrm{Ni}(1 \mu \mathrm{g} / \mathrm{side} ; n=7)$, or c-PTIO ( 1 $\mu \mathrm{g} / \mathrm{side} ; n=7$ ), then sacrificed $2 \mathrm{~h}$ and $40 \mathrm{~min}$ following infusion. (G) Representative blots for Arc/Arg3.1, c-Fos, EGR-1, and GAPDH in the LA. (H) Representative blots for Arc/Arg3.1, c-Fos, EGR-1, and GAPDH in the MGm/PIN. (I) Mean ( \pm SEM) percent Arc/Arg3.1, c-Fos, and EGR-1 immunoreactivity from LA punches taken from naïve rats given intra-LA infusions of vehicle, Ifenprodil, 7-Ni, or c-PTIO. Here, protein levels have been normalized to GAPDH levels for each sample. ( $/$ ) Mean ( \pm SEM) percent Arc/Arg3.1, c-Fos, and EGR-1 immunoreactivity from MGm/PIN punches taken from naïve rats given intra-LA infusions of vehicle, Ifenprodil, 7-Ni, or c-PTIO. Here, protein levels have been normalized to GAPDH levels for each sample. $(*) P<0.05$ relative to vehicle-infused rats.

Representative Western blots from either LA or MGm/PIN for rats given intra-LA infusion of Rp-8-Br-PET-cGMPS can be viewed in Figure 5, B and C, respectively. Relative to vehicle-infused controls, rats given intra-LA infusion of Rp-8-Br-PET-cGMPS prior to training exhibited significant decreases in levels of Arc/Arg3.1 $\left(t_{(22)}=3.29, P<0.01\right)$, c-Fos $\left(t_{(22)}=2.72, P<0.05\right)$, and EGR-1 $\left(t_{(15)}=2.75, P<0.05\right)$ protein in the LA (Fig. 5D). In the MGm/ PIN, we found similar significant decreases in levels of Arc/ Arg3.1 $\left(t_{(20)}=2.33, P<0.05\right)$, c-Fos $\left(t_{(19)}=3.07, P<0.01\right)$, and EGR-1 $\left(t_{(20)}=3.24, P<0.01\right)$ protein (Fig. 5E). Importantly, levels of the loading control, GAPDH, did not differ between the vehicle- and Rp-8-Br-PET-cGMPS-infused rats in either the LA or MGm/PIN samples.

Representative Western blots from either LA or MGm/PIN for rats given intra-LA infusion of 8-Br-cGMP can be viewed in Figure 6, B and $\mathrm{C}$, respectively. Relative to vehicle-infused controls, rats given intra-LA infusion of 8 -Br-cGMP prior to training exhibited significant increases in levels of Arc/Arg3.1 $\left(t_{(20)}=2.94, P<0.01\right)$, c-Fos $\left(t_{(20)}=2.27, P<0.05\right)$, and EGR-1 $\left(t_{(20)}=3.01, P<0.01\right)$ protein in the LA (Fig. 6D). In the MGm/ PIN, we found similar significant increases in levels of Arc/ Arg3.1 $\left(t_{(24)}=3.21, P<0.01\right)$, c-Fos $\left(t_{(27)}=3.32, P<0.01\right)$, and EGR-1 $\left(t_{(26)}=3.03, P<0.01\right)$ proteins (Fig. 6E). Levels of the loading control, GAPDH, however, did not differ between the vehicle- and 8-Br-cGMP-infused rats in either the LA or MGm/ PIN samples.

Importantly, this reduction or enhancement in IEG expression in both the LA and MGm/PIN following intra-LA infusion of Rp-8-Br-PET-cGMPS or 8-Br-cGMP, respectively, was not observed in naïve animals that did not receive fear conditioning (Figs. 5F-J, $6 \mathrm{~F}-\mathrm{J}$ ). Representative Western blots from either LA or MGm/PIN for naïve rats given intra-LA infusion of Rp-8-Br-PET-cGMPS can be viewed in Figure 5, G and H, respectively, and representative Western blots from either LA or MGm/PIN for naïve rats given intra-LA infusion of 8-Br-cGMP can be viewed in Figure 6, G and $\mathrm{H}$, respectively. Relative to vehicle controls, naïve rats given intra-LA infusion of either Rp-8-Br-PET-cGMPS or 8-Br-cGMP prior to sacrifice at the same time as the trained animals described above exhibited no significant differences in levels of these IEGS in either the LA or the MGm/PIN (Figs. 5I,J, 6I,J). In addition, levels of the loading control, GAPDH, did not differ between any of the groups in either the LA or the MGm/PIN.

To further support our findings that each of the drugs that we used in our experiments have affected training-induced changes in IEG expression, we performed an additional analysis for each of our drug groups comparing both conditioned and naïve 

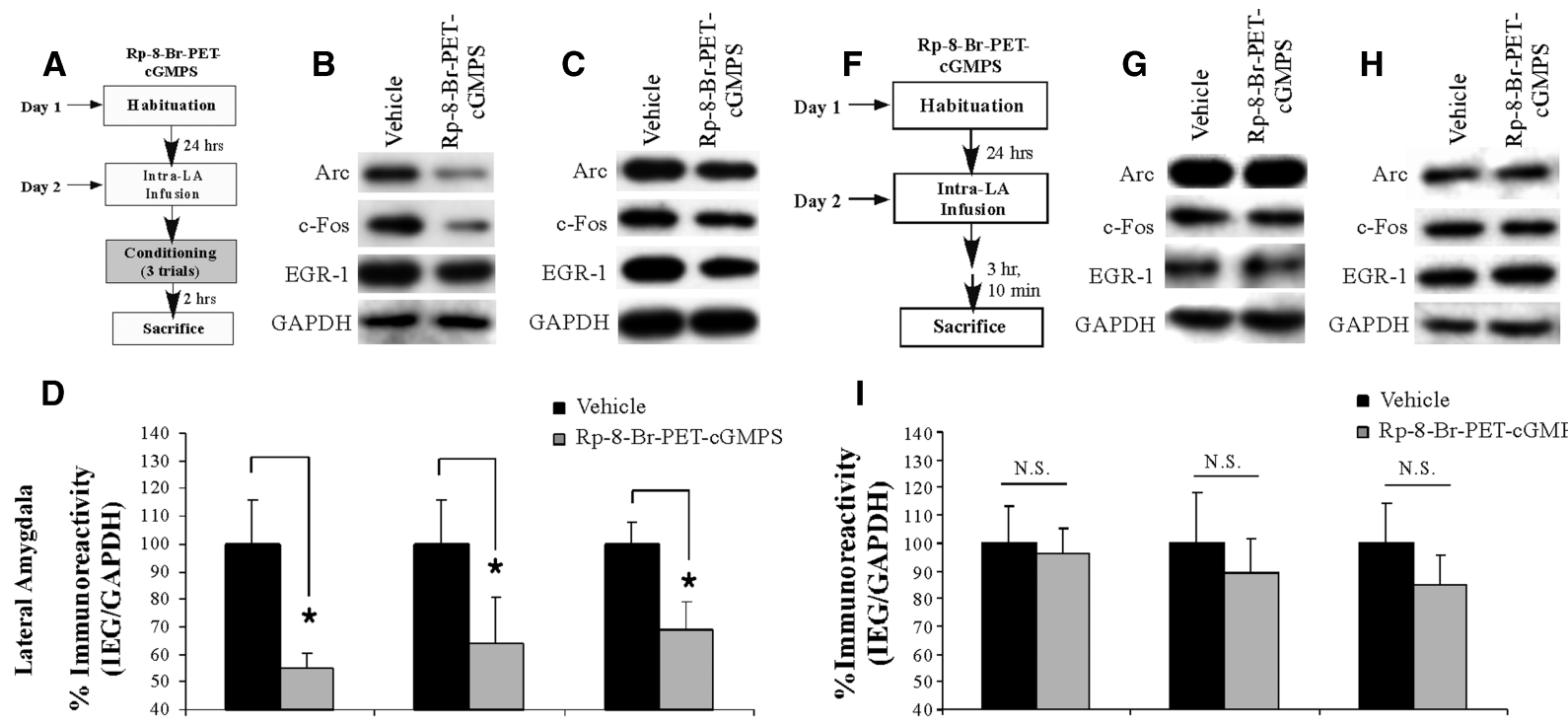

E
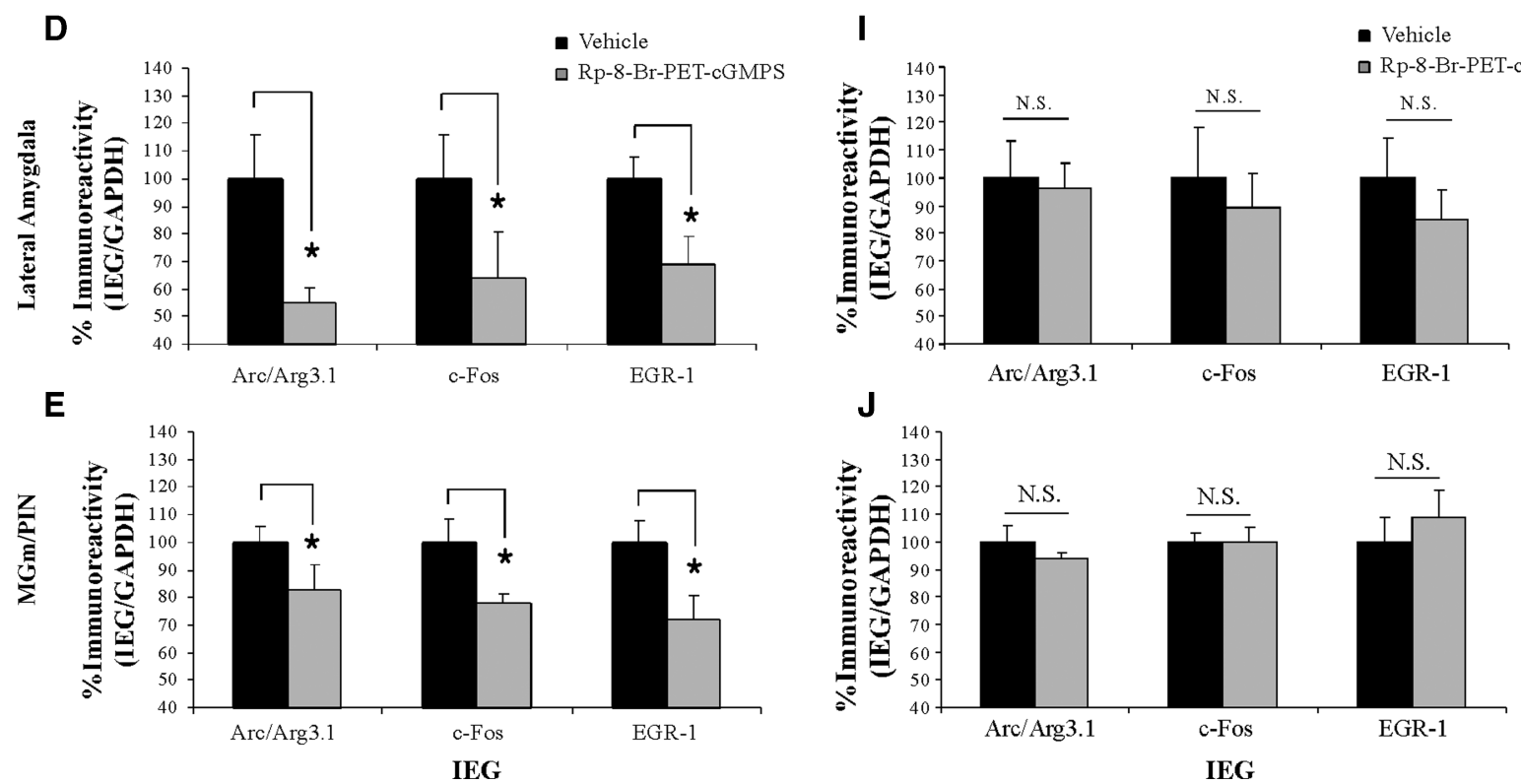

Figure 5. Intra-LA infusion of a PKG inhibitor impairs ERK-driven gene expression in the LA and MGm/PIN following fear conditioning. (A) Schematic of behavioral protocol for the PKG inhibitor. Rats were given intra-LA infusion of the vehicle or Rp-8-Br-PET-cGMPS (1 $\mu \mathrm{g} /$ side), followed $1 \mathrm{~h}$ later by fear conditioning. Rats were sacrificed $2 \mathrm{~h}$ following training. (B) Representative blots for Arc/Arg3.1, c-Fos, EGR-1, and GAPDH in the LA. (C) Representative blots for Arc/Arg3.1, c-Fos, EGR-1, and GAPDH in the MGm/PIN. (D) Mean ( \pm SEM) percent Arc/Arg3.1, c-Fos, and EGR-1 immunoreactivity from LA punches taken from rats given intra-LA infusions of vehicle (Arc/Arg3.1: $n=10$, c-Fos: $n=10$, EGR-1: $n=7$ ) or Rp-8-Br-PET-cGMPS (Arc/Arg3.1: $n=14$, c-Fos: $n=14$, EGR-1: $n=10$ ). Here, Arc/Arg3.1, c-Fos, and EGR-1 levels have been normalized to GAPDH levels for each sample. (E) Mean ( \pm SEM) percent Arc/Arg3.1, c-Fos, and EGR-1 immunoreactivity from MGm/PIN punches taken from rats in D given intra-LA infusions of vehicle (Arc/Arg3.1: $n=9$, c-Fos: $n=9$, EGR-1: $n=9$ ) or Rp-8-Br-PET-cGMPS (Arc/Arg3.1: $n=13$, c-Fos: $n=12$, EGR-1: $n=13$ ). Here, Arc/Arg3.1, c-Fos, and EGR-1 levels have been normalized to GAPDH levels for each sample. $(F)$ Schematic of behavioral protocol. Rats were given intra-LA infusion of the vehicle or Rp-8-Br-PET-cGMPS $(1 \mu \mathrm{g} /$ side). Rats were sacrificed $3 \mathrm{~h}$ and $10 \mathrm{~min}$ following infusion. (G) Representative blots for Arc/Arg3.1, c-Fos, EGR-1, and GAPDH in the LA. (H) Representative blots for Arc/Arg3.1, c-Fos, EGR-1, and GAPDH in the MGm/PIN. (I) Mean ( \pm SEM) percent Arc/Arg3.1, c-Fos, and EGR-1 immunoreactivity from LA punches taken from naïve rats given intra-LA infusions of vehicle (Arc/Arg 3.1: $n=5$, C-Fos: $n=5$, EGR-1: $n=5$ ) or Rp-8-Br-PET-cGMPS (Arc/Arg3.1: $n=5$, c-Fos: $n=5$, EGR-1: $n=4$ ). Here, protein levels have been normalized to GAPDH levels for each sample. (J) Mean ( \pm SEM) percent Arc/Arg3.1, c-Fos, and EGR-1 immunoreactivity from MGm/PIN punches taken from naïve rats given intra-LA infusions of vehicle (Arc/Arg3.1: $n=5$, c-Fos: $n=5$, EGR-1: $n=5$ ) or Rp-8-Br-PET-cGMPS (Arc/Arg3.1: $n=5$, c-Fos: $n=5$, EGR-1: $n=5$ ). Here, protein levels have been normalized to GAPDH levels for each sample. $\left({ }^{*}\right) P<0.05$ relative to vehicle-infused rats.

animals within the same overall Drug Condition (Vehicle vs. Drug) by Training Condition (Naïve vs. Trained) by IEG (Arc/ Arg3.1 vs. EGR-1 vs. c-Fos) ANOVA. In these experiments, all animals were treated identically except for the presence or absence of paired tone-shock presentations (i.e., both Naïve and Trained groups were each infused with the same dose and volume of drug and sacrificed at the same time point following infusion of the drug). Here, we report only the results of the Drug Condition (Vehicle vs. Drug) by Training Condition (Naïve vs. Trained) interaction, since this analysis is the most relevant for supporting our conclusion that our effects on IEG expression in both the LA and MGm/PIN are specific to training and not due to a generalized effect of infusion of the drugs alone. Further, we found no significant effects for the Drug Condition by IEG, Training Condition by IEG, or Drug Condition by Training Condition by IEG interactions in any of our analyses.
The overall ANOVA for Naïve and Trained animals infused with Ifenprodil showed a significant Drug Condition by Training Condition interaction in both the LA $\left(F_{(1,71)}=9.79\right.$, $P<0.01)$ and the MGm/PIN $\left(F_{(1,78)}=16.43, P<0.001\right)$. A similar pattern of results was seen for 7-Ni-infused animals. The ANOVA showed a significant Drug Condition by Training Condition interaction in both the LA $\left(F_{(1,74)}=6.07, P<0.05\right)$ and the MGm/PIN $\left(F_{(1,75)}=12.77, P<0.001\right)$. The ANOVA for c-PTIO-infused animals, in contrast, showed a nonsignificant Drug Condition by Training Condition interaction in the LA $\left(F_{(1,73)}=0.52, P>\right.$ $0.05)$, but a significant Drug Condition by Training Condition interaction in the MGm/PIN $\left(F_{(1,78)}=18.43, P<0.001\right)$. This is consistent with our earlier analysis, in which we showed that animals given intra-LA infusion of c-PTIO showed significant differences relative to the vehicle group in expression of the IEGs Arc/Arg3.1, c-Fos, and EGR-1 in the MGm/PIN (Fig. 4E), but 

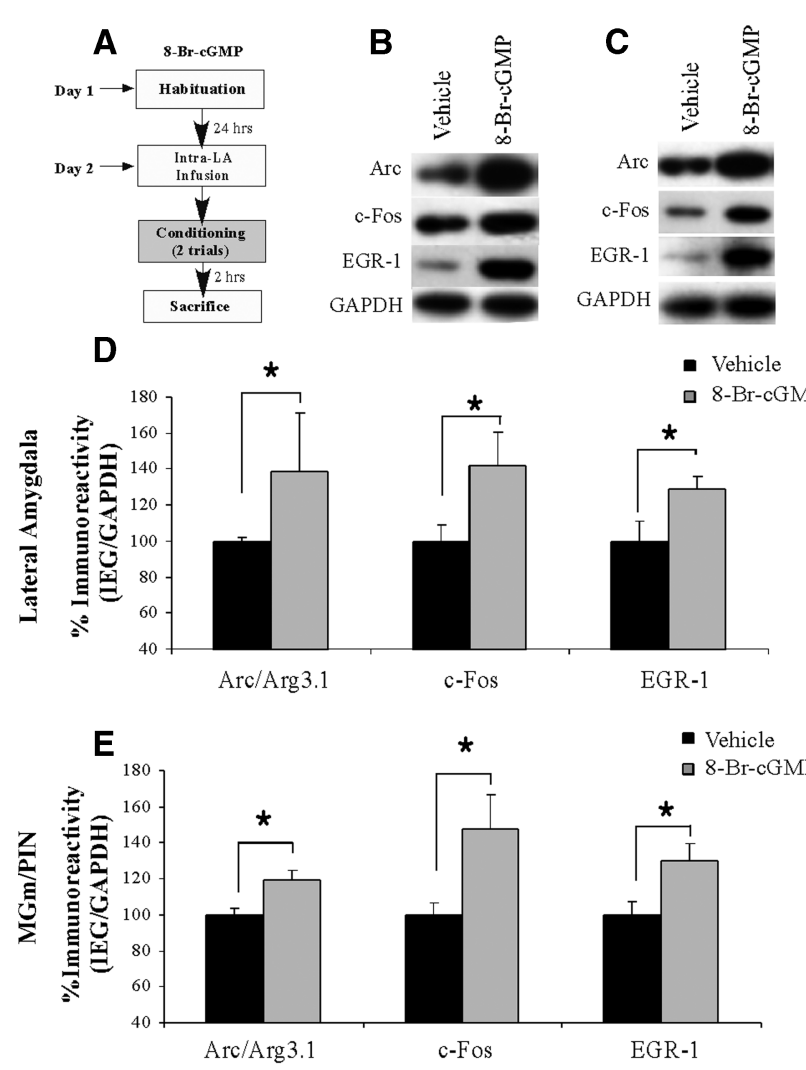

IEG

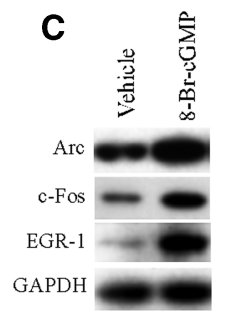

- Vehicle - 8-Br-cGMP

$\star$

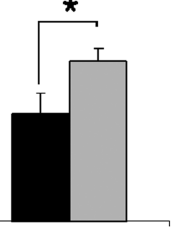

EGR-1
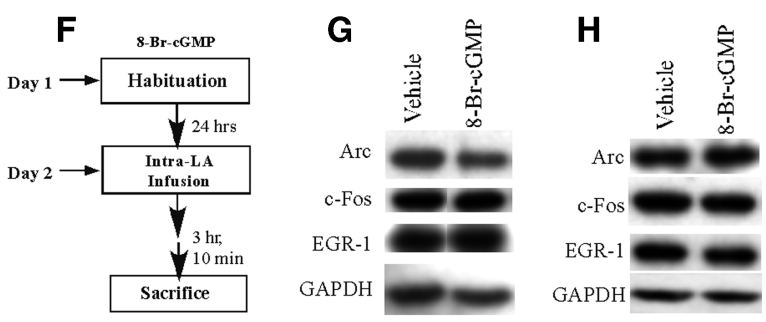

I
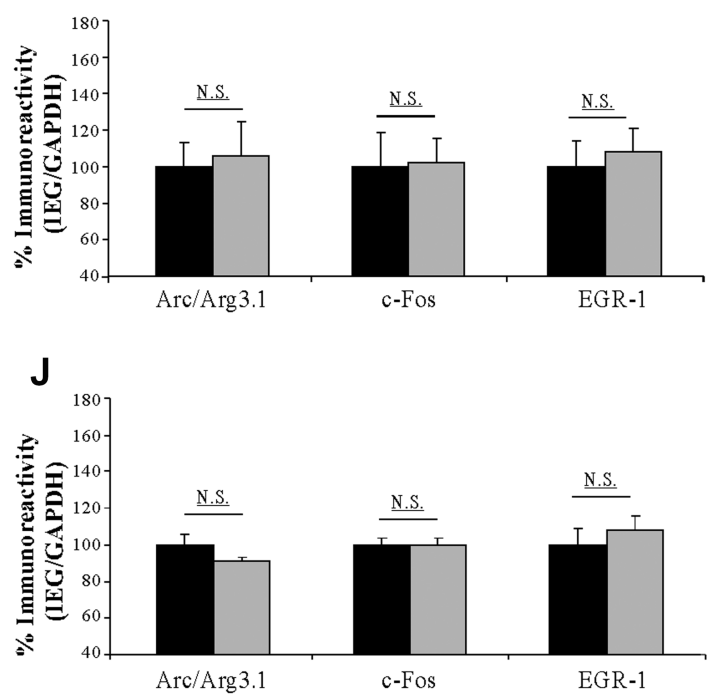

IEG

Figure 6. Intra-LA infusion of a PKG activator enhances ERK-driven gene expression in the LA and MGm/PIN following fear conditioning. ( $A$ ) Schematic of behavioral protocol for PKG activator. Rats were given intra-LA infusion of the vehicle or 8-Br-cGMP (10 $\mu \mathrm{g} / \mathrm{side})$, followed $1 \mathrm{~h}$ later by fear conditioning. Rats were sacrificed $2 \mathrm{~h}$ following training. (B) Representative blots for Arc/Arg3.1, c-Fos, EGR-1, and GAPDH in the LA. (C) Representative blots for Arc/Arg3.1, c-Fos, EGR-1, and GAPDH in the MGm/PIN. (D) Mean ( \pm SEM) percent Arc/Arg3.1, c-Fos, and EGR-1 immunoreactivity from LA punches taken from rats given intra-LA infusions of vehicle (Arc/Arg3.1: $n=11$, c-Fos: $n=11$, EGR-1: $n=11)$ or 8-Br-cGMP (Arc/Arg3.1: $n=11, \mathrm{c}-$ Fos: $n=11$, EGR-1: $n=11)$. Here, Arc/Arg3.1, c-Fos, and EGR-1 levels have been normalized to GAPDH levels for each sample. (E) Mean ( \pm SEM) percent Arc/Arg3.1, c-Fos, and EGR-1 immunoreactivity from MGm/PIN punches taken from rats in $D$ given intra-LA infusions of vehicle (Arc/Arg3.1: $n=13, \mathrm{c}-$ Fos: $n=15$, EGR-1: $n=14$ ) or 8-Br-cGMP (Arc/Arg3.1: $n=13$, c-Fos: $n=14$, EGR-1: $n=14$ ). Here, Arc/Arg3.1, c-Fos, and EGR-1 levels have been normalized to GAPDH levels for each sample. $(F)$ Schematic of behavioral protocol. Rats were given intra-LA infusion of the vehicle or 8 -Br-cGMP (10 $\mu$ g/side). Rats were sacrificed $3 \mathrm{~h}$ and $10 \mathrm{~min}$ following infusion. (G) Representative blots for Arc/Arg3.1, C-Fos, EGR-1, and GAPDH in the LA. (H) Representative blots for Arc/Arg3.1, c-Fos, EGR-1, and GAPDH in the MGm/PIN. (I) Mean ( \pm SEM) percent Arc/Arg3.1, c-Fos, and EGR-1 immunoreactivity from LA punches taken from naïve rats given intra-LA infusions of vehicle (Arc/Arg3.1: $n=5, \mathrm{c}-\mathrm{Fos}: n=5, \mathrm{EGR}-1: n=5$ ) or 8-Br-cGMP (Arc/Arg3.1: $n=6$, c-Fos: $n=6$, EGR-1: $n=6$ ). Here, protein levels have been normalized to GAPDH levels for each sample. $(J)$ Mean ( \pm SEM) percent Arc/Arg3.1, C-Fos, and EGR-1 immunoreactivity from MGm/PIN punches taken from naïve rats given intra-LA infusions of vehicle (Arc/Arg3.1: $n=5, \mathrm{c}-$ Fos: $n=5$, EGR-1: $n=5$ ) or 8-Br-cGMP (Arc/Arg3.1: $n=4$, c-Fos: $n=6$, EGR-1: $n=6$ ). Here, protein levels have been normalized to GAPDH levels for each sample. $\left(^{*}\right) P<0.05$ relative to vehicle-infused rats.

not in the LA (Fig. 4D). The ANOVA for the Rp-8-Br-PET-cGMPSinfused animals showed a significant Drug Condition by Training Condition interaction in both the LA $\left(F_{(1,82)}=5.48\right.$, $P<0.05)$ and in the $\mathrm{MGm} / \mathrm{PIN}\left(F_{(1,83)}=10.50, P<0.01\right)$. Similarly, the ANOVA for 8-Br-cGMP-infused animals showed a significant Drug Condition by Training Condition interaction in both the LA $\left(F_{(1,87)}=5.21, P<0.05\right)$ and in the MGm/PIN $\left(F_{(1,102)}=9.76, P<0.01\right)$. Taken together, the results of these analyses suggest that our effects on IEG expression in both the LA and MGm/PIN are not due to a generalized effect of infusion of the drugs alone. Rather, our effects appear to be specific to the combination of training with the drug infused.

Collectively, the findings from this second series of experiments suggest that NMDAR-driven synaptic plasticity and NO-cGMP-PKG signaling in the LA regulate fear memory consolidation via training-induced expression of the IEGs Arc/Arg3.1, c-Fos, and EGR-1 in both the LA and in the MGm/PIN. Further, given the fact that the NOS inhibitor 7-Ni impairs traininginduced IEG expression in both the LA and the MGm/PIN, while the membrane impermeable NO scavenger c-PTIO selectively impairs training-induced IEG expression in the MGm/PIN, these findings further suggest that synaptic plasticity in the LA during fear conditioning promotes IEG expression in MGm/PIN neurons via extracellular release of NO from LA neurons.

\section{Training-induced IEG expression in the LA and MGm/PIN is downstream from ERK/MAPK activation}

The experiments presented thus far support the hypothesis that NMDAR-driven synaptic plasticity and NO-cGMP-PKG signaling in the LA at the time of fear learning coordinately regulate ERK and ERK-driven transcriptional changes in MGm/PIN and LA neurons. In our first series of experiments, we showed that pharmacological manipulations in the LA that target synaptic plasticity and 
the NO-cGMP-PKG signaling pathway regulate ERK/MAPK phosphorylation in the LA and MGm/PIN (Figs. 1-3). In our second series of experiments, we showed that NMDAR-driven synaptic plasticity and NO-cGMP-PKG signaling in the LA also regulate the expression of the IEGs Arc/Arg3.1, c-Fos, and EGR-1 in the LA and the MGm/PIN (Figs. 46). In this final set of experiments, we ask whether these two processes are linked: that is, whether the training induced expression of IEGs that we have observed in the LA and MGm/PIN are downstream from the ERK/MAPK signaling pathway.

\section{Inhibition of ERK/MAPK signaling in the LA impairs training-induced IEG expression in the $L A$, but not in the MGm/PIN}

In this first experiment, we gave rats intra-LA infusion of either vehicle or the MEK inhibitor U0126 ( $1 \mu \mathrm{g} / \mathrm{side}$; $0.5 \mu \mathrm{L}$ ), a dose that we have shown to effectively impair fear memory consolidation when infused into the LA (Schafe et al. 2000). Thirty minutes later, rats were conditioned using three toneshock pairings as before and sacrificed $2 \mathrm{~h}$ later. Western blotting was used to assay for the IEGs Arc/Arg3.1, c-Fos, and EGR-1 in both the LA and MGm/ PIN (Fig. 7A). Relative to vehicle-infused controls, rats given intra-LA infusion of U0126 exhibited significant decreases in the expression of Arc/Arg3.1 $\left(t_{(13)}=\right.$ $2.49, P<0.05)$, c-Fos $\left(t_{(13)}=3.96, P<\right.$ $0.01)$, and EGR-1 $\left(t_{(13)}=2.82, P<0.05\right)$ protein in the LA (Fig. 7B). In the MGm/PIN, however, no significant differences in levels of Arc/Arg3.1, c-Fos, or EGR-1 protein were observed (Fig. 7C). Representative blots for each IEG from the LA and MGm/PIN are represented adjacent to Figure 7, B and $\mathrm{C}$, respectively. Importantly, levels of the loading control, GAPDH, did not differ between vehicle- and U0126-infused rats in either the LA or the MGm/PIN. Thus, intra-LA infusion of U0126 impairs IEG expression in the LA, but not in the MGm/PIN.
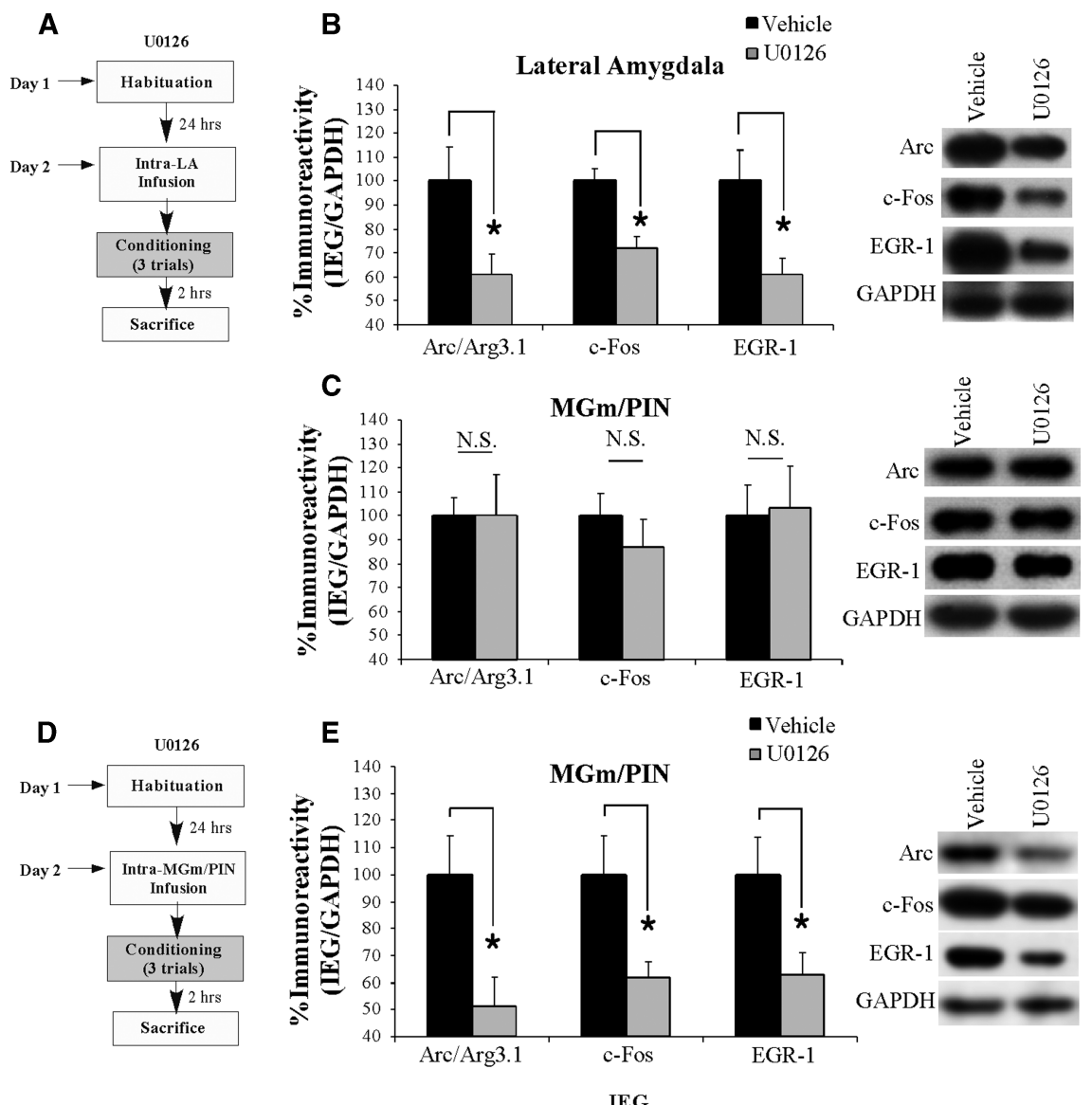

Figure 7. Training-induced IEG expression in the LA and MGm/PIN is downstream from ERK/MAPK in the LA and MGm/PIN, respectively. $(A)$ Schematic of behavioral protocol for intra-LA infusion of U0126. Rats were given intra-LA infusion of the vehicle or U0126 (1 $\mu \mathrm{g} / \mathrm{side})$, followed $30 \mathrm{~min}$ later by fear conditioning. Rats were sacrificed $2 \mathrm{~h}$ following training. (B) Mean ( \pm SEM) percent Arc/ Arg3.1, c-Fos, and EGR-1 immunoreactivity from LA punches taken from rats given intra-LA infusions of vehicle (Arc/Arg3.1: $n=7$, c-Fos: $n=7$, EGR-1: $n=7$ ) or U0126 (Arc/Arg3.1: $n=8$, c-Fos: $n=8$, EGR-1: $n=8$ ). Here, Arc/Arg3.1, c-Fos, and EGR-1 levels have been normalized to GAPDH levels for each sample. Representative blots for Arc/Arg3.1, c-Fos, EGR-1, and GAPDH can be viewed at the right. (C) Mean ( \pm SEM) percent Arc/Arg3.1, c-Fos, and EGR-1 immunoreactivity from MGm/PIN punches taken from rats in $B$ given intra-LA infusions of vehicle (Arc/Arg3.1: $n=9$, c-Fos: $n=9$, EGR-1: $n=9$ ) or U0126 (Arc/Arg3.1: $n=8$, c-Fos: $n=8$, EGR-1: $n=8$ ). Here, Arc/Arg3.1, c-Fos, and EGR-1 levels have been normalized to GAPDH levels for each sample. Representative blots for Arc/Arg3.1, c-Fos, EGR-1, and GAPDH can be viewed at the right. (D) Schematic of behavioral protocol for intra-MGm/PIN infusion of U0126. Rats were given intra-MGm/PIN infusion of vehicle or U0126 (1 $\mu \mathrm{g} /$ side), followed $30 \mathrm{~min}$ later by fear conditioning. Rats were sacrificed $2 \mathrm{~h}$ following training. (E) Mean ( \pm SEM) percent Arc/Arg3.1, c-Fos, and EGR-1 immunoreactivity from MGm/PIN punches taken from rats given intra-MGm/PIN infusions of vehicle (Arc/Arg3.1: $n=5$, c-Fos: $n=5, \mathrm{EGR}-1: n$ $=5$ ) or U0126 (Arc/Arg3.1: $n=7$, c-Fos: $n=7$, EGR-1: $n=7$ ). Here, Arc/Arg3.1, c-Fos, and EGR-1 levels have been normalized to GAPDH levels for each sample. Representative blots for Arc/Arg3.1, c-Fos, EGR-1, and GAPDH can be viewed at the right. $\left(^{*}\right) P<0.05$ relative to vehicle-infused rats.

\section{Inhibition of ERK/MAPK signaling in the MGm/}

\section{PIN impairs training-induced IEG expression in the MGm/PIN}

Next, we asked whether training-induced IEG expression in the $\mathrm{MGm} / \mathrm{PIN}$ is downstream from ERK/MAPK signaling in the $\mathrm{MGm} / \mathrm{PIN}$. We gave rats intra-MGm/PIN infusion of either vehicle or U0126 $(1 \mu \mathrm{g} /$ side; $0.5 \mu \mathrm{L})$, a dose that we have shown to effectively block fear memory consolidation when infused into the MGm/PIN (Apergis-Schoute et al. 2005). Thirty minutes later, rats were conditioned using three tone-shock pairings as before and sacrificed $2 \mathrm{~h}$ later. Punches taken from the MGm/PIN were homogenized and probed for Arc/Arg3.1, c-Fos, and EGR-1 (Fig. 7D). Relative to vehicle-infused controls, rats given an intra-MGm/PIN infusion of U0126 exhibited significant decreases in levels of Arc/Arg3.1 $\left(t_{(10)}=2.74, P<0.05\right)$, c-Fos $\left(t_{(10)}=2.75, P<0.05\right)$, and EGR-1 $\left(t_{(10)}=2.48, P<0.05\right)$ protein in the MGm/PIN (Fig. 7E). Representative blots for each IEG from the $\mathrm{MGm} / \mathrm{PIN}$ are represented adjacent to Figure 7E. Importantly, levels of the loading control, GAPDH, did not differ between the vehicle- and U0126-infused rats for Arc/Arg3.1, c-Fos, or EGR-1, indicating that overall protein levels were not significantly changed in animals that received U0126. These findings indicate that IEG expression in the MGm/PIN is downstream from ERK/MAPK, suggesting that the mechanism of IEG regulation there may be similar to that in the LA. 


\section{Discussion}

Recent studies from our laboratory have shown that blockade or facilitation of the NO-cGMP-PKG signaling pathway in the LA impairs or enhances memory consolidation of Pavlovian fear conditioning and synaptic plasticity in the LA by activating the ERK/MAPK signaling pathway (Schafe et al. 2005b; Ota et al. 2008). Further, we and others have shown that ERK-driven gene expression in the MGm/PIN plays a critical role in fear memory consolidation (Apergis-Schoute et al. 2005) and synaptic plasticity in the LA (Apergis-Schoute et al. 2005). In the present study, we now show that pharmacological manipulation of synaptic plasticity and NO-cGMP-PKG signaling in the LA regulates the expression of ERK and ERK-driven IEGs in both the LA and the MGm/PIN following auditory fear conditioning. These findings suggest that NMDAR-driven synaptic plasticity and the NO-cGMP-PKG signaling pathway may promote fear memory consolidation, in part, by regulating ERK-driven transcription at both sides of the thalamo-LA synapse.

\section{NO signaling promotes ERK-driven gene expression in both the $L A$ and $M G m / P I N$}

In both vertebrate and invertebrate models of memory formation, long-term synaptic plasticity is thought to involve NMDARdriven recruitment of intracellular signaling pathways that promote long-term plastic change and memory through alterations of transcription and translation and accompanying morphological changes at both pre- and postsynaptic sites (Bonhoeffer et al. 1989; Lisman and Harris 1993; Engert and Bonhoeffer 1999; Malenka and Nicoll 1999; Toni et al. 1999; Roberts and Glanzman 2003). Further, many studies have suggested that the NO-cGMP-PKG signaling pathway plays a critical role in coordinating these two events (Schuman and Madison 1991; Zhuo et al. 1994; Arancio et al. 1996; Son et al. 1998; Lu et al. 1999; Monfort et al. 2002). In the postsynaptic cell, for example, NMDAR-driven activation of NOS and the formation of NO has been suggested to play a critical role in transcriptional regulation (Lu et al. 1999), while, presynaptically, activation of cGMPPKG signaling via "retrograde signaling" of NO has been suggested to promote mobilization of synaptic vesicles in the presynaptic cell, leading to enhanced transmitter release (Ninan et al. 2006) as well as structural changes in the presynaptic terminal (Antonova et al. 2001; Wang et al. 2005). In our experiments, we targeted the NO-cGMP-PKG signaling pathway in the LA at several different levels. Of particular interest is the finding that intra-LA infusion of the NOS inhibitor $7-\mathrm{Ni}$ or the PKG inhibitor Rp-8-Br-PET-cGMPS impairs ERK activation and ERK-driven gene expression in both the LA and MGm/PIN, while intra-LA infusion of the membrane impermeable NO scavenger c-PTIO impairs ERK activation and ERK-driven gene expression in the MGm/PIN, but not in the LA. These findings indicate that ERK-driven gene expression at both sides of the thalamo-LA synapse is coordinately regulated by NO-cGMP-PKG signaling in the LA, and are consistent with the view that NO-driven "retrograde signaling" in the LA during fear conditioning promotes ERK activation and ERK-driven transcription in MGm/PIN neurons.

\section{A revised model of fear memory consolidation}

The present findings are consistent with, and expand upon a revised model of fear learning and memory consolidation in which NMDAR-driven synaptic plasticity and NO signaling in LA neurons promotes pre- and postsynaptic aspects of fear memory formation at thalamo-LA synapses via regulation of ERK-driven gene expression in MGm/PIN and LA neurons, respectively (Fig. 8). In that model, fear memory consolidation is hypothesized to involve both pre- and postsynaptic modifications at thalamo-LA synapses. These modifications are first triggered by NMDAR-mediated activation of the NO-cGMP-PKG signaling pathway in the postsynaptic cell (Step 1) that promotes the activation of ERK (Schafe et al. 2000) (Step 2) and ERK-driven transcription (Bailey et al. 1999; Josselyn et al. 2001) in LA neurons (Step 3). The transcription of these ERK-driven genes is ultimately thought to lead to postsynaptic functional and/or structural changes that contribute to the formation of the memory (Rodrigues et al. 2004a). Concurrently, "retrograde signaling"

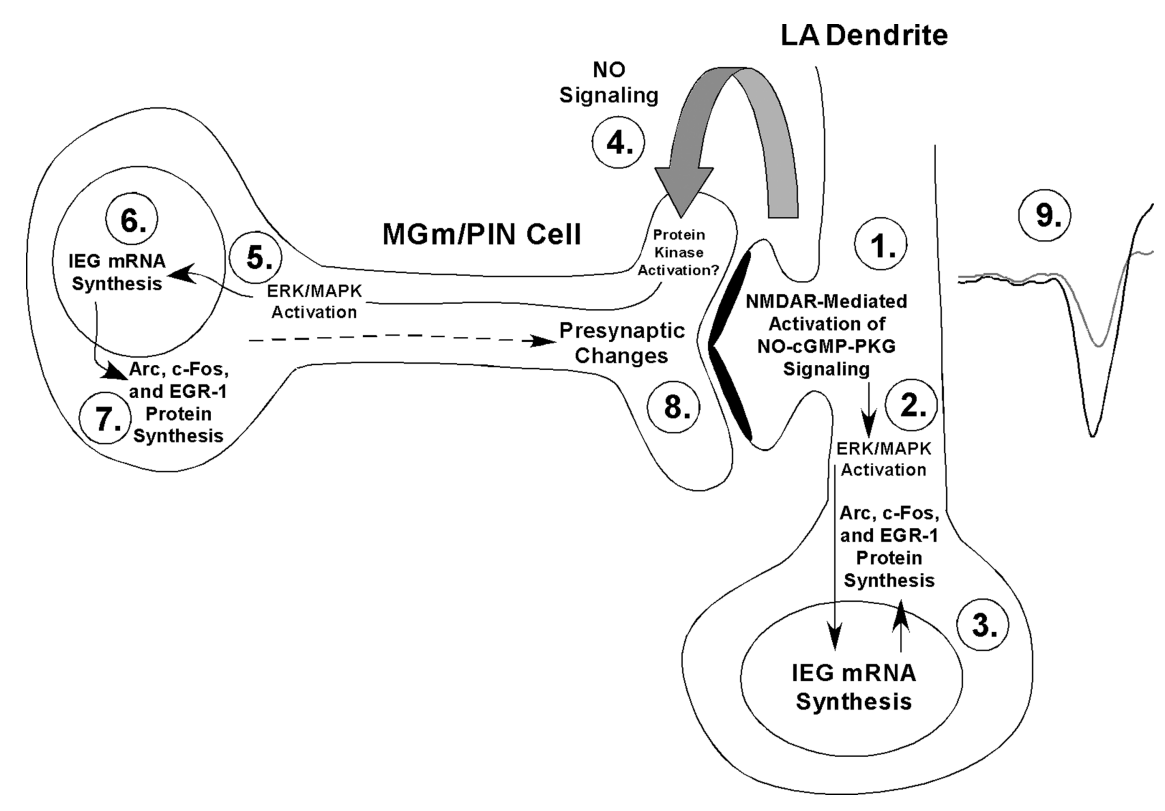

Figure 8. A model of fear memory consolidation. Fear memory consolidation is hypothesized to involve both pre- and postsynaptic modifications at thalamo-LA synapses. These modifications are first triggered by NMDAR-mediated alterations in protein kinase signaling pathways in LA neurons (Step 1) that promote ERK-dependent signaling in LA neurons (Step 2). Signaling via ERK/MAPK in LA neurons, in turn, promotes the transcription of IEGs including Arc/Arg3.1, c-Fos, and EGR-1 (Step $3)$, which is ultimately thought to lead to postsynaptic functional and/or structural changes that contribute to the formation of the memory. Concurrently, NMDAR-driven synaptic plasticity in LA neurons is also hypothesized to lead to the activation of nNOS in LA neurons and the release of nitric oxide (NO; Step 4), which can, in turn, engage ERK-driven signaling (Step 5) and mRNA synthesis (Step 6), followed by translation of IEGs including Arc/Arg3.1, c-Fos, and EGR-1 in MGm/PIN neurons (Step 7). ERK-driven gene expression in MGm/PIN neurons is, in turn, hypothesized to promote presynaptic functional and/or structural changes at thalamo-LA synapses (Step 8). Together with the postsynaptic modifications driven by ERK signaling in the LA, these presynaptic modifications act to strengthen the connectivity of thalamo-LA synapses, which is reflected neurophysiologically in an enhanced response to the CS in the LA after training (Step 9). 
via NO (Step 4) may promote the activation of ERK (Step 5) and ERK-driven transcription (Steps 6,7) in presynaptic thalamic targets of LA neurons that are necessary to promote structural and/ or functional changes on the presynaptic side of LA synapses (Step 8). Together with the postsynaptic modifications driven by ERK signaling in the LA, these presynaptic modifications act to strengthen the connectivity of thalamo-LA synapses, which is reflected neurophysiologically in an enhanced response to the CS in the LA after training (Step 9). Importantly, previous studies from our laboratory and others have shown that ERK-driven transcription in the $\mathrm{MGm} / \mathrm{PIN}$ is required not only for fear memory consolidation (Apergis-Schoute et al. 2005; Parsons et al. 2006; Han et al. 2008), but also for synaptic plasticity at thalamo-LA synapses (Apergis-Schoute et al. 2005). Further, auditory fear conditioning has recently been shown to lead to increased expression of the presynaptically localized protein synaptophysin in the LA (Nithianantharajah and Murphy 2008), suggesting that fear memory consolidation is accompanied by presynaptic alterations at LA synapses. Finally, a recent study from our laboratory showed that fear conditioning is associated with increases in the ERK-driven IEG EGR-1 in the MGm/PIN, and that knockdown of EGR-1 in MGm/PIN neurons impairs both fear memory consolidation and the training-induced expression of both synapsin and synaptophysin in the LA (Overeem et al. 2010). Together with the findings of the present study, these findings collectively suggest that synaptic plasticity and NO signaling in the LA regulate ERK and ERK-driven gene expression in the MGm/PIN that contributes to fear memory consolidation by promoting presynaptic aspects of plasticity at the level of the LA.

\section{Two pools of ERK activation associated with fear memory formation}

In the present study, we found that although pharmacological inhibition of synaptic plasticity (via Ifenprodil) or NO signaling (via 7-Ni, c-PTIO, or Rp-8-Br-PET-cGMPS) in the LA impairs ERK-driven gene expression in the MGm/PIN, inhibition of ERK/MAPK (via U0126) in the LA has no effect at the thalamic level (Fig. 7C). In contrast, we found that intra-MGm/PIN infusion of U0126 does in fact impair ERK-driven gene expression in the MGm/PIN (Fig. 7E). This pattern of findings suggests that there are two functionally distinct pools of ERK/MAPK, one in LA neurons and one in MGm/PIN neurons, that are required for fear memory consolidation (Schafe et al. 2000; Apergis-Schoute et al. 2005). Further, our findings suggest that each pool of ERK is linked by a common upstream mechanism (e.g., NO-cGMP-PKG signaling) in the LA. While the mechanism by which NO signaling leads to ERK activation in the LA during fear learning is presently unknown, previous studies have suggested that PKG or its downstream substrates can activate Raf-1, an upstream regulator of ERK1/2 (Hood and Granger 1998), or inhibit protein phosphatase-1 (Hall et al. 1998), which may indirectly regulate ERK1/2.

A second outstanding question is how NO signaling at the level of the LA might be regulating ERK and ERK-driven gene expression at the thalamic level. Our revised model of fear memory consolidation hypothesizes that "retrograde" signaling via $\mathrm{NO}$ at thalamo-LA synapses promotes the activation of a presynaptic target that, via some retrograde transport process, leads to ERK-driven gene expression at the somatic level in MGm/PIN neurons ( $\sim 2.5 \mathrm{~mm}$ away; Fig. 8). Previous reports have suggested that such retrograde transport can occur very rapidly in neurons, between $\sim 4$ and $8 \mathrm{~mm} / \mathrm{hr}$ (Oztas 2003). Our findings, however, suggest that this retrogradely transported presynaptic target can- not be ERK itself; intra-LA infusion of the MEK inhibitor U0126, which would presumably affect ERK activation both pre- and postsynaptically, has no effect on training-induced elevations of Arc/Arg3.1, c-Fos, or EGR-1 in the MGm/PIN (Fig. 7C). Two additional candidates are PKG and $\alpha$ CaMKII. In in vitro models of synaptic plasticity in the hippocampus, both PKG and $\alpha$ CaMKII are known presynaptic targets of NO. Presynaptic injection of an $\alpha$ CaMKII inhibitor peptide, for example, has been shown to block both LTP at CA1 synapses and accompanying presynaptic morphological alterations induced by NO donors or cGMP analogs (Ninan and Arancio 2004), suggesting that $\alpha$ CaMKII may act downstream from cGMP-PKG signaling to promote presynaptic aspects of plasticity. Our laboratory has recently shown that intra-LA infusion of either an inhibitor or activator of cGMP-PKG signaling impairs or enhances, respectively, synaptic plasticity at thalamo-LA synapses, fear memory consolidation, and ERK activation in the LA (Ota et al. 2008). Similarly, fear conditioning has been shown to regulate the autophosphorylation of $\alpha$ CaMKII at postsynaptic sites, while inhibition of CaMKII activity in the LA impairs fear memory formation and synaptic plasticity at thalamo-LA synapses (Rodrigues et al. 2004b). While these findings suggest that signaling via both PKG and $\alpha$ CaMKII are critical regulators of postsynaptic signaling in LA neurons (Rodrigues et al. 2004b; Ota et al. 2008), these same pathways may also be critical for regulating ERK and ERK-driven gene expression in the MGm/PIN following fear learning. Future experiments will be necessary to further define the signaling pathways by which NO regulates ERK in the MGm/PIN during fear learning.

In the present study, we have used pharmacological tools to define the contribution of NMDAR-driven synaptic plasticity and NO-cGMP-PKG signaling in the LA on ERK activation and ERK-driven gene expression in both the LA and the MGm/ PIN. Given that many of our pharmacological manipulations have similar effects in both LA and MGm/PIN, it is critical to address the possibility that passive drug diffusion from the LA to the MGm/PIN can account for our findings. However, we think this is an unlikely possibility. For example, we show by using intra-LA infusion of two different drugs (c-PTIO; U0126) that we can obtain differential effects on molecular signaling cascades in LA and MGm/PIN. This is particularly striking in the case of U0126, which is capable of blocking IEG expression in either the LA or MGm/PIN when infused directly into either structure. However, when U0126 is infused into LA, it impairs IEG expression in LA, while sparing that in MGm/PIN (Fig. 7). Further, a recent study from our laboratory showed that infusion of an antisense oligodeoxynucleotide to EGR-1 that remains confined to the MGm/PIN impairs fear memory consolidation and associated presynaptic alterations at the level of the LA (Overeem et al. 2010). Collectively, we think these observations provide the best evidence that passive drug diffusion between the LA and the MGm/PIN is unlikely to account for our findings.

In summary, the results of the present study clearly suggest that synaptic plasticity and the NO-cGMP-PKG signaling pathway in the LA coordinately regulate ERK and ERK-driven gene expression in the LA and in the MGm/PIN, helping to further define a signaling cascade involved in the formation of emotional memories in the LA and further supporting a revised model of fear learning that incorporates a role for the auditory thalamus. The present findings also provide further support for the role of NO as a "retrograde messenger" in the LA, and make an additional contribution toward understanding the cellular and molecular processes underlying emotional memory formation in the amygdala. 


\section{Materials and Methods}

\section{Subjects}

Adult male Sprague-Dawley rats (Harlan) were housed individually in plastic cages and maintained on a 12:12 h light/dark cycle. Food and water were provided ad libitum throughout the experiment.

\section{Surgical procedures}

Under a mixture of Ketamine $(100 \mathrm{mg} / \mathrm{kg})$ and Xylazine $(6.0 \mathrm{mg} /$ $\mathrm{kg}$ ) anesthesia, rats were implanted bilaterally with 22- or 26-gauge stainless steel guide cannulas (Plastics One) aimed at the LA or MGm/PIN, respectively. The coordinates for the LA were: $-3.2 \mathrm{~mm}, \pm 5.0 \mathrm{~mm},-8.0 \mathrm{~mm}$ relative to bregma. The coordinates for the MGm/PIN were: $-5.5 \mathrm{~mm}, \pm 2.8 \mathrm{~mm}$, $-6.6 \mathrm{~mm}$ relative to bregma. The guide cannulas were fixed to screws in the skull using a mixture of acrylic and dental cement, and a 28- or 31-gauge dummy cannula was inserted into each guide cannula to prevent clogging. Rats were given Buprenex $(0.2 \mathrm{mg} / \mathrm{kg})$ as an analgesic and given at least $5 \mathrm{~d}$ to recover prior to experimental procedures. All procedures were conducted in accordance with the National Institutes of Health Guide for the Care and Use of Experimental Animals and were approved by the Yale University Animal Care and Use Committee.

\section{Drugs}

The NR2B selective antagonist $\alpha$-(4-Hydroxyphenyl)- $\beta$-methyl-4benzyl-1-piperidineethanol (+)-tartrate salt (Ifenprodil; Sigma, Cat. No. 12892) was dissolved in physiological saline at a concentration of $2 \mu \mathrm{g} / \mu \mathrm{L}$ in $2 \%$ HBC. The NOS inhibitor 7-Nitroindazole, 3-Bromo-, sodium salt (7-Ni; EMD Chemicals, Cat. No. 483400), the membrane impermeable NO scavenger 2-(4-Carboxyphenyl)-4,4,5,5-tetramethylimidazoline-1-oxyl-3oxide, potassium salt (c-PTIO; Tocris, Cat. No. 0772), and the MEK inhibitor 1,4-Diamino-2,3-dicyano-1,4-bis(aminophenylthio)butadiene (U0126; Promega, Cat. No. V1121) were dissolved in $100 \%$ DMSO to yield a stock concentration of $4 \mu \mathrm{g} / \mu \mathrm{L}$, which was then diluted 1:1 in ACSF prior to infusion. The PKG inhibitor Guanosine 3',5'-cyclic Monophosphorothioate, b-Phenyl-1, $\mathrm{N}^{2}$ etheno-8-bromo-, Rp-Isomer, sodium salt (Rp-8-Br-PET-cGMPS; Calbiochem, Cat. No. 370679) and the PKG activator Guanosine 3',5'-cyclic Monophosphate, 8-Bromo-, sodium salt (8-Br-cGMP; Calbiochem, Cat. No. 203820) were dissolved in distilled water at concentrations of either $2 \mu \mathrm{g} / \mu \mathrm{L}$ (Rp-8-Br-PET-cGMPS) or $20 \mu \mathrm{g} / \mu \mathrm{L}(8-\mathrm{Br}-\mathrm{cGMP})$.

\section{Behavioral procedures}

Rats were habituated to handling and dummy cannula removal for $2 \mathrm{~d}$ prior to training. On the training day, rats were given intra-LA infusion of 50\% DMSO in ACSF (vehicle), the NR2B selective antagonist Ifenprodil $(1 \mu \mathrm{g} /$ side in $0.5 \mu \mathrm{L} ; 0.25 \mu \mathrm{L} / \mathrm{min})$, the NOS inhibitor 7-Ni $(1 \mu \mathrm{g} /$ side in $0.5 \mu \mathrm{L} ; 0.25 \mu \mathrm{L} / \mathrm{min})$, the membrane impermeable NO scavenger c-PTIO $(1 \mu \mathrm{g} /$ side in $0.5 \mu \mathrm{L}$; $0.25 \mu \mathrm{L} / \mathrm{min})$, or the MEK inhibitor U0126 $(1 \mu \mathrm{g} /$ side in $0.5 \mu \mathrm{L}$; $0.25 \mu \mathrm{L} / \mathrm{min})$. In other experiments, rats were given an intra-LA infusion of either ACSF (vehicle; containing [in millimolars]: $115 \mathrm{NaCl}, 3.3 \mathrm{KCl}, 1 \mathrm{MgSO}_{4}, 2 \mathrm{CaCl}_{2}, 25.5 \mathrm{NaHCO}_{3}, 1.2$ $\mathrm{NaH}_{2} \mathrm{PO}_{4}$, and 10 glucose), the PKG inhibitor Rp-8-Br-PETcGMPS $(1 \mu \mathrm{g} /$ side in $0.5 \mu \mathrm{L} ; 0.25 \mu \mathrm{L} / \mathrm{min})$, or the PKG activator 8-Br-cGMP $(10 \mu \mathrm{g} /$ side in $0.5 \mu \mathrm{L} ; 0.25 \mu \mathrm{L} / \mathrm{min})$. In a final set of experiments, rats received intra-MGm/PIN infusion of vehicle (50\% DMSO in ACSF) or U0126 $(1 \mu \mathrm{g} /$ side in $0.5 \mu \mathrm{L} ; 0.25 \mu \mathrm{L} /$ $\mathrm{min})$. Injectors remained in the cannulas for 1 min after drug infusion to allow diffusion of the drug from the tip.

Thirty (Ifenprodil, 7-Ni, c-PTIO, and U0126, and respective vehicle controls) or 60 (Rp-8-Br-PET-cGMPS and 8-Br-cGMP, and respective vehicle controls) min following drug infusion, rats were trained with three conditioning trials consisting of a 20- sec, $5-\mathrm{kHz}, 75-\mathrm{dB}$ tone that coterminated with a $1.0-\mathrm{sec}, 1-\mathrm{mA}$ footshock (intertrial interval [ITI] $=120 \mathrm{sec}$ ). For experiments involving intra-LA infusion of 8-Br-cGMPS, rats received two tone-shock pairings consisting of a $20-\mathrm{sec}, 5-\mathrm{kHz}, 75-\mathrm{dB}$ tone that coterminated with a $1.0-\mathrm{sec}, 0.5-\mathrm{mA}$ footshock (ITI $=120$ $\mathrm{sec})$. This slightly weaker training protocol was used in an effort to avoid ceiling effects that might obscure observation of training-induced elevations in ERK and ERK-driven IEGs above the level of vehicle controls (Ota et al. 2008). Either 1 or $2 \mathrm{~h}$ following training, rats received an overdose of chloral hydrate $(250 \mathrm{mg} / \mathrm{kg})$ and were decapitated. The 1-h time point has previously been shown to be optimal for assaying training-induced elevations in ERK/MAPK activation in the LA (Schafe et al. 2000; Ota et al. 2008). The 2-h time point has also previously been shown to be optimal for assaying training-induced elevations in IEG activation in the LA and the MGm/PIN (Ploski et al. 2008).

\section{Western blotting}

Following behavioral training and sacrifice, brains were frozen and stored at $-80^{\circ} \mathrm{C}$ until processed. Punches containing the LA or the MGm/PIN were obtained using a 1-mm punch tool (Fine Science Tools) from $400-\mu \mathrm{m}$-thick sections taken on a sliding freezing microtome. Representative punches from both LA and MGm/PIN can be viewed in Supplemental Figure 2. Punches were manually dounced in $100 \mu \mathrm{L}$ of ice-cold hypotonic lysate buffer (10 mM Tris-Hcl, pH 7.5, $1 \mathrm{mM}$ EDTA, $2.5 \mathrm{mM}$ sodium pyrophosphate, $1 \mathrm{mM}$ phenylmethylsulfonyl fluoride, $1 \mathrm{mM}$ $\beta$-glycero-phosphate, 1\% Igepal CA-630, 1\% protease inhibitor cocktail [Sigma], and $1 \mathrm{mM}$ sodium orthovanadate). Sample buffer $(25 \mu \mathrm{L})$ was immediately added to the homogenates, and the samples were boiled for $4 \mathrm{~min}$. Homogenates were electrophoresed on $10 \%$ Tris- $\mathrm{HCl}$ gels and blotted to Immobilon-P (Millipore). Western blots were blocked in 5\% milk in TTBS buffer (50 mM Tris-Hcl, pH 7.5, $150 \mathrm{mM} \mathrm{NaCl}$, and $0.05 \%$ Tween $20)$, then incubated with anti-phospho-MAPK (1:1000; Cell Signaling), anti-total MAPK (1:1000; Cell Signaling), anti-Arc (1:1000; Santa Cruz), anti-c-Fos (1:1000; Santa Cruz), or antiEGR-1 (1:1000; Santa Cruz) antibody. Blots were then incubated with the appropriate secondary antibody conjugated to horseradish peroxidase (1:20K; Cell Signaling) and developed using enhanced chemiluminescence (Pierce). GAPDH (1:5000; Abcam) was used as a loading control for all Western blotting experiments to control for inconsistencies in protein loading. Optical densities of the bands were analyzed using NIH Image J software.

To assess for changes in the activation of ERK/MAPK, total ERK levels were first normalized to the reference protein GAPDH for each sample. Activated (phosphorylated) ERK levels were then normalized to total ERK. For analysis, activated ERK levels in drug-infused rats were expressed as a percentage of those in vehicle-infused rats.

\section{Immunohistochemistry}

For immunohistochemistry experiments, rats were cannulated as described above. Rats were habituated to handling and dummy cannula removal for $2 \mathrm{~d}$ prior to training. On the training day, animals were given an intra-LA infusion of either ACSF (vehicle), the PKG inhibitor Rp-8-Br-PET-cGMPS $(1 \mu \mathrm{g} /$ side in $0.5 \mu \mathrm{L}$; $0.25 \mu \mathrm{L} / \mathrm{min})$ or the PKG activator 8 -Br-cGMP $(10 \mu \mathrm{g} /$ side in $0.5 \mu \mathrm{L} ; 0.25 \mu \mathrm{L} / \mathrm{min})$. Injectors remained in the cannulas for $1 \mathrm{~min}$ after drug infusion to allow diffusion of the drug from the tip. One hour following drug infusion, rats were trained with either two (8-Br-cGMP) or three (Rp-8-Br-PET-cGMPS) conditioning trials consisting of a $20-\mathrm{sec}, 5-\mathrm{kHz}, 75-\mathrm{dB}$ tone that coterminated with a $1.0-\mathrm{sec}, 0.5-\mathrm{mA}$ or $1-\mathrm{mA}$ footshock, respectively $(\mathrm{ITI}=120 \mathrm{sec})$. One hour following training, rats were rapidly and deeply anesthetized with chloral hydrate $(250 \mathrm{mg} / \mathrm{kg}$, i.p.) and perfused through the heart with ice-cold phosphate-buffered saline (PBS), followed by ice-cold $4 \%$ paraformaldehyde in $0.1 \mathrm{M}$ phosphate buffer $(\mathrm{PB})$. Following perfusion, brains were removed 
and post-fixed in $4 \%$ paraformaldehyde-PB for $12 \mathrm{~h}$ and then cryoprotected in $20 \%$ glycerol- $0.1 \mathrm{M}$ PB for $48-72 \mathrm{~h}$. Free-floating sections $(40 \mu \mathrm{m})$ containing either the LA or the MGm/PIN were cut using a sliding microtome. After blocking in Tris-buffered saline (TBS) containing $1 \%$ goat serum- $0.1 \%$ Triton X-100, slices were incubated overnight at room temperature in anti-phosphoMAPK antibody (1:1000; Cell Signaling) in TBS-1\% goat serum$0.1 \%$ Triton X-100. After three washes in PBS, tissue sections were visualized using VectaStain $\mathrm{ABC}$ kit (Vector Laboratories) and developed using a DAB peroxidase substrate (Sigma). Sections were mounted on Fisherbrand electrostatic slides and coverslipped.

\section{Acknowledgments}

This research was supported by the National Science Foundation (NSF 0444632 to G.E.S.), the National Institutes of Health (MH 073949 to G.E.S.), and by Yale University. K.T.O. is supported by a National Science Foundation Graduate Research Fellowship.

\section{References}

Antonova I, Arancio O, Trillat AC, Wang HG, Zablow L, Udo H, Kandel ER, Hawkins RD. 2001. Rapid increase in clusters of presynaptic proteins at onset of long-lasting potentiation. Science 294: 1547-1550.

Apergis-Schoute AM, Debiec J, Doyere V, LeDoux JE, Schafe GE. 2005. Auditory fear conditioning and long-term potentiation in the lateral amygdala require ERK/MAP kinase signaling in the auditory thalamus: A role for presynaptic plasticity in the fear system. J Neurosci 25: 5730-5739.

Arancio O, Kiebler M, Lee CJ, Lev-Ram V, Tsien RY, Kandel ER, Hawkins RD. 1996. Nitric oxide acts directly in the presynaptic neuron to produce long-term potentiation in cultured hippocampal neurons. Cell 87: $1025-1035$.

Bailey DJ, Kim JJ, Sun W, Thompson RF, Helmstetter FJ. 1999. Acquisition of fear conditioning in rats requires the synthesis of mRNA in the amygdala. Behav Neurosci 113: 276-282.

Blair HT, Schafe GE, Bauer EP, Rodrigues SM, LeDoux JE. 2001. Synaptic plasticity in the lateral amygdala: A cellular hypothesis of fear conditioning. Learn Mem 8: 229-242.

Bonhoeffer T, Staiger V, Aertsen A. 1989. Synaptic plasticity in rat hippocampal slice cultures: Local "Hebbian" conjunction of pre- and postsynaptic stimulation leads to distributed synaptic enhancement. Proc Natl Acad Sci 86: 8113-8117.

Chien WL, Liang KC, Teng CM, Kuo SC, Lee FY, Fu WM. 2003. Enhancement of long-term potentiation by a potent nitric oxideguanylyl cyclase activator, 3-(5-hydroxymethyl-2-furyl)-1-benzylindazole. Mol Pharmacol 63: 1322-1328.

Engert F, Bonhoeffer T. 1999. Dendritic spine changes associated with hippocampal long-term synaptic plasticity. Nature 399: 66-70.

Hall KU, Collins SP, Gamm DM, Massa E, DePaoli-Roach AA, Uhler MD. 1998. Phosphorylation-dependent inhibition of protein phosphatase-1 by G-substrate. J Biol Chem 274: 3485-3495.

Hall J, Thomas KL, Everitt BJ. 2001. Fear memory retrieval induces CREB phosphorylation and Fos expression within the amygdala. Eur $J$ Neurosci 13: $1453-1458$.

Han JH, Yiu AP, Cole CJ, Hsiang HL, Neve RL, Josselyn SA. 2008. Increasing CREB in the auditory thalamus enhances memory and generalization of auditory conditioned fear. Learn Mem 15: 443-453.

Hood J, Granger HJ. 1998. Protein kinase G mediates vascular endothelial growth factor-induced Raf- 1 activation and proliferation in human endothelial cells. J Biol Chem 273: 23504-23508.

Huff NC, Frank M, Wright-Hardesty K, Sprunger D, Matus-Amat P, Higgins E, Rudy JW. 2006. Amygdala regulation of immediate-early gene expression in the hippocampus induced by contextual fear conditioning. J Neurosci 26: 1616-1623.

Josselyn SA, Shi C, Carlezon WA, Neve RL, Nestler EJ, Davis M. 2001. Longterm memory is facilitated by cAMP response element-binding protein overexpression in the amygdala. J Neurosci 21: 2404-2412.

Ko SW, Ao HS, Mendel AG, Qiu CS, Wei F, Milbrandt J, Zhuo M. 2005. Transcription factor Egr-1 is required for long-term fear memory and anxiety. Sheng Li Xue Bao 57: 421-432.

Lander HM, Ogiste JS, Pearce SF, Levi R, Novogrodsky A. 1995. Nitric oxidestimulated guanine nucleotide exchange on pp21ras. J Biol Chem 270: 7017-7020.

Lander HM, Jacovina AT, Davis RJ, Tauras JM. 1996. Differential activation of mitogen-activated protein kinases by nitric oxide-related species. $J$ Biol Chem 271: 19705-19709.
Lander HM, Hajjar DP, Hempstead BL, Mirza UA, Chait BT, Campbell S, Quilliam LA. 1997. A molecular redox switch on pp21(ras). Structural basis for the nitric oxide-p21(ras) interaction. J Biol Chem 272: $4323-4326$.

Lin CH, Yeh SH, Lu HY, Gean PW. 2003. The similarities and diversities of signal pathways leading to consolidation of conditioning and consolidation of extinction of fear memory. J Neurosci 23: 8310-8317.

Lisman JE, Harris KM. 1993. Quantal analysis and synaptic anatomyintegrating two views of hippocampal plasticity. Trends Neurosci 16: $141-147$.

Lu YF, Kandel ER, Hawkins RD. 1999. Nitric oxide signaling contributes to late-phase LTP and CREB phosphorylation in the hippocampus. $J$ Neurosci 19: 10250-10261.

Malenka RC, Nicoll RA. 1999. Long-term potentiation-a decade of progress? Science 285: 1870-1874.

Malkani S, Rosen JB. 2000. Specific induction of early growth response gene 1 in the lateral nucleus of the amygdala following contextual fear conditioning in rats. Neuroscience 97: 693-702.

Malkani S, Wallace KJ, Donley MP, Rosen JB. 2004. An egr-1 (zif268) antisense oligodeoxynucleotide infused into the amygdala disrupts fear conditioning. Learn Mem 11: 617-624.

Monfort P, Munoz MD, Kosenko E, Felipo V. 2002. Long-term potentiation in hippocampus involves sequential activation of soluble guanylate cyclase, cGMP-dependent protein kinase, and cGMP-degrading phosphodiesterase. J Neurosci 22: 10116-10122.

Ninan I, Arancio O. 2004. Presynaptic CaMKII is necessary for synaptic plasticity in cultured hippocampal neurons. Neuron 42: 129-141.

Ninan I, Liu S, Rabinowitz D, Arancio O. 2006. Early presynaptic changes during plasticity in cultured hippocampal neurons. Embo J 25: $4361-4371$.

Nithianantharajah J, Murphy M. 2008. Auditory specific fear conditioning results in increased levels of synaptophysin in the basolateral amygdala. Neurobiol Learn Mem 90: 36-43.

Ota KT, Pierre VJ, Ploski JE, Queen K, Schafe GE. 2008. The NO-cGMP-PKG signaling pathway regulates synaptic plasticity and fear memory consolidation in the lateral amygdala via activation of ERK/MAP kinase. Learn Mem 15: 792-805.

Overeem KA, Ota KT, Monsey MS, Ploski JE, Schafe GE. 2010. A role for nitric oxide-driven retrograde signaling in the consolidation of a fear memory. Front Behav Neurosci 4: 2. doi: 10.3389/neuro.08.002.2010.

Oztas E. 2003. Neuronal tracing. Neuroanatomy 2: 2-5.

Parsons RG, Riedner BA, Gafford GM, Helmstetter FJ. 2006. The formation of auditory fear memory requires the synthesis of protein and mRNA in the auditory thalamus. Neuroscience 141: 1163-1170.

Paul S, Olausson P, Venkitaramani DV, Ruchkina I, Moran TD, Tronson N, Mills E, Hakim S, Salter MW, Taylor JR, et al. 2007. The striatalenriched protein tyrosine phosphatase gates long-term potentiation and fear memory in the lateral amygdala. Biol Psychiatry 61: 1049-1061.

Ploski JE, Pierre VJ, Smucny J, Park K, Monsey MS, Overeem KA, Schafe GE 2008. The activity-regulated cytoskeletal-associated protein (Arc/ Arg3.1) is required for memory consolidation of Pavlovian fear conditioning in the lateral amygdala. J Neurosci 28: 12383-12395.

Roberts AC, Glanzman DL. 2003. Learning in Aplysia: Looking at synaptic plasticity from both sides. Trends Neurosci 26: 662-670.

Rodrigues SM, Schafe GE, LeDoux JE. 2001. Intra-amygdala blockade of the NR2B subunit of the NMDA receptor disrupts the acquisition but not the expression of fear conditioning. J Neurosci 21: 6889-6896.

Rodrigues SM, Schafe GE, LeDoux JE. 2004a. Molecular mechanisms underlying emotional learning and memory in the lateral amygdala. Neuron 44: 75-91.

Rodrigues SM, Farb CR, Bauer EP, LeDoux JE, Schafe GE. 2004b. Pavlovian fear conditioning regulates Thr286 autophosphorylation of $\mathrm{Ca}^{2+} /$ calmodulin-dependent protein kinase II at lateral amygdala synapses. J Neurosci 24: 3281-3288.

Rosen JB, Fanselow MS, Young SL, Sitcoske M, Maren S. 1998. Immediateearly gene expression in the amygdala following footshock stress and contextual fear conditioning. Brain Res 796: $132-142$.

Schafe GE, Atkins CM, Swank MW, Bauer EP, Sweatt JD, LeDoux JE. 2000. Activation of ERK/MAP kinase in the amygdala is required for memory consolidation of Pavlovian fear conditioning. J Neurosci 20: 8177-8187.

Schafe GE, Nader K, Blair HT, LeDoux JE. 2001. Memory consolidation of Pavlovian fear conditioning: A cellular and molecular perspective. Trends Neurosci 24: 540-546.

Schafe GE, Doyere V, LeDoux JE. 2005a. Tracking the fear engram: The lateral amygdala is an essential locus of fear memory storage. J Neurosci 25: $10010-10014$.

Schafe GE, Bauer EP, Rosis S, Farb CR, Rodrigues SM, LeDoux JE. 2005b. Memory consolidation of Pavlovian fear conditioning requires nitric oxide signaling in the lateral amygdala. Eur J Neurosci 22: 201-211. 
Schuman EM, Madison DV. 1991. A requirement for the intercellular messenger nitric oxide in long-term potentiation. Science 254: $1503-1506$.

Son H, Lu YF, Zhuo M, Arancio O, Kandel ER, Hawkins RD. 1998. The specific role of cGMP in hippocampal LTP. Learn Mem 5: $231-245$.

Toni N, Buchs PA, Nikonenko I, Bron CR, Muller D. 1999. LTP promotes formation of multiple spine synapses between a single axon terminal and a dendrite. Nature 402: 421-425.

Wallace CS, Lyford GL, Worley PF, Steward O. 1998. Differential intracellular sorting of immediate early gene mRNAs depends on signals in the mRNA sequence. $J$ Neurosci 18: 26-35.

Wang HG, Lu FM, Jin I, Udo H, Kandel ER, de Vente J, Walter U, Lohmann SM, Hawkins RD, Antonova I. 2005. Presynaptic and postsynaptic roles of $\mathrm{NO}, \mathrm{cGK}$, and RhoA in long-lasting potentiation and aggregation of synaptic proteins. Neuron 45: 389-403.
Yun HY, Gonzalez-Zulueta M, Dawson VL, Dawson TM. 1998. Nitric oxide mediates $N$-methyl-D-aspartate receptor-induced activation of pp21ras. Proc Natl Acad Sci 95: 5773-5778.

Yun HY, Dawson VL, Dawson TM. 1999. Glutamate-stimulated calcium activation of Ras/Erk pathway mediated by nitric oxide. Diabetes Res Clin Pract 45: $113-115$.

Zhang WP, Guzowski JF, Thomas SA. 2005. Mapping neuronal activation and the influence of adrenergic signaling during contextual memory retrieval. Learn Mem 12: 239-247.

Zhuo M, Hu Y, Schultz C, Kandel ER, Hawkins RD. 1994. Role of guanylyl cyclase and cGMP-dependent protein kinase in long-term potentiation. Nature 368: 635-639.

Received August 13, 2009; accepted in revised form February 12, 2010. 


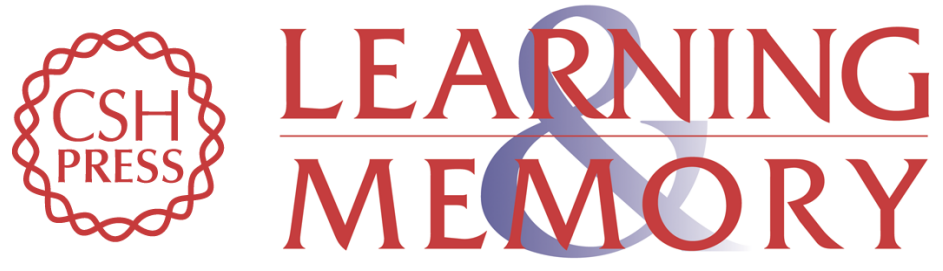

\section{Synaptic plasticity and NO-cGMP-PKG signaling coordinately regulate ERK-driven gene expression in the lateral amygdala and in the auditory thalamus following Pavlovian fear conditioning}

Kristie T. Ota, Melissa S. Monsey, Melissa S. Wu, et al.

Learn. Mem. 2010, 17:

Access the most recent version at doi:10.1101/lm.1592510

Supplemental Material

References License

Email Alerting Service
http://learnmem.cshlp.org/content/suppl/2010/03/29/17.4.221.DC1

This article cites 51 articles, 28 of which can be accessed free at: http://learnmem.cshlp.org/content/17/4/221.full.html\#ref-list-1

Receive free email alerts when new articles cite this article - sign up in the box at the top right corner of the article or click here. 